This manuscript is a non-peer reviewed EarthArXiv preprint that has been submitted for publication in Ocean Modelling. If accepted, the final version of this manuscript will be available via the 'Peer-reviewed Publication DOI' link on the right-hand side of this webpage. 


\title{
Optimal experiment design for bottom friction parameter estimation
}

\author{
Simon C. Warder ${ }^{\mathrm{a}, *}$, Matthew D. Piggott ${ }^{\mathrm{a}}$ \\ ${ }^{a}$ Department of Earth Science and Engineering, Imperial College London, London, SW7 2AZ, UK
}

\begin{abstract}
It is common practice within numerical coastal ocean modelling to perform model calibration with respect to a bottom friction parameter. While many modelling studies employ a spatially uniform coefficient, within the parameter estimation literature the coefficient is typically taken to be spatially (or even temporally) varying. A parameter estimation experiment requires an appropriate set of observations, and also the selection of an appropriate parameter space which captures the spatial variability of the bottom friction parameter. In regions such as the Bristol Channel, which is used as a case study within this work, observation data is relatively abundant; here we use observations of M2 and S2 harmonic amplitudes and phases at 20 locations within the Channel. However, as is typical within friction parameter estimation problems, there is no obvious constraint on the spatial variation of the friction coefficient. Here, we define the parameter estimation 'experiment design' as the mapping from a small number of friction parameters onto the model domain. We propose a robust method for the appropriate selection of a low-dimensional experiment design, utilising an optimal experiment design (OED) technique via construction of the Fisher Information Matrix. The objective is to identify the experiment design resulting in the tightest possible constraints on the unknown parameters, given the available observation data. We construct the Fisher Information Matrix via the use of an adjoint shallow water numerical model, Thetis, and perform a variant of D-optimal design to find the optimal experiment design from within two a priori choices of design space. These are based on splitting the model domain either by simple slices across the channel, or by the type of sediment found on the sea bed. We first validate the OED framework by utilising a Bayesian inference algorithm to perform parameter estimation using a selection of experiment designs, which confirms that the OED framework offers a good estimate of the true parameter uncertainty resulting from the use of a given experiment design. An exploration of the full space of experiment designs shows that up to three Manning's $n$ coefficient values can be estimated from the observation data to within an uncertainty of approximately $0.001 \mathrm{~s} \mathrm{~m}^{-1 / 3}$, but that the experiment design is highly influential in achieving this threshold, thus demonstrating the value of our approach as a preliminary step in a parameter estimation study. We also investigate the sensitivity of the achievable parameter uncertainty to the availability of observation data and its measurement uncertainty, providing insights useful to the design of future observation surveys. Finally, we further demonstrate our OED framework with an application to a model of the northwest European continental shelf.
\end{abstract}

Keywords: Optimal experiment design, Fisher Information Matrix, Parameter estimation, Bottom friction, Manning coefficient

\footnotetext{
* Corresponding author

Email addresses: s.warder15@imperial.ac.uk (Simon C. Warder), m.d.piggott@imperial.ac.uk (Matthew D. Piggott)
} 


\section{Introduction}

Numerical tidal models are utilised within a wide range of application areas, including sediment and pollutant transport (e.g. Periáñez et al. (2013), Chen and Liu (2017), Li et al. (2018)), ecosystems and fisheries (e.g. Marshall et al. (2017), Whomersley et al. (2018)) and marine renewable energy (e.g. Adcock et al. (2015), Neill et al. (2018), Mackie et al. (2020a), Wang and Yang (2020)), as well as underpinning the modelling of coastal hazards including storm surges (Flather, 2000). Accurate tidal models are therefore of significant value, yet any model output is subject to a variety of sources of uncertainty.

Within coastal ocean modelling, a significant source of uncertainty is the parameterisation of bottom friction. Friction between the ocean and the sea bed arises due to a boundary layer at the interface, and due to form drag induced by bathymetry undulations. The resulting momentum loss is not explicitly captured in coastal ocean models, but is instead typically incorporated via a parameterised drag term in the governing equations, which relates the water velocity to a frictional force. There are many possible choices of drag parameterisation (Zhang et al., 2011, Döös et al., 2004), each of which introduces a drag parameter. Furthermore, this parameter in principle varies spatially, due to variations in sea bed roughness and hydrodynamic conditions, as well as potential dependence on model mesh resolution given that the parameterisation represents subgrid-scale processes. Even when it can be related to land or sea floor classification using well-established tables, this parameter still carries significant uncertainty (Mayo et al., 2014). The bottom friction coefficient may also vary over time, e.g. due to morphological changes at the sea bed (Davies and Robins, 2017) or seasonal variations in hydrological conditions (Huybrechts et al., 2021). However, since the coefficient's value cannot be directly measured in the field, this parameter is a source of significant uncertainty within coastal ocean models.

For this reason, it is common within the coastal ocean modelling literature to perform model calibration with respect to the bottom friction coefficient, whereby its value is inferred from observations of hydrodynamic variables. Commonly used observations for friction parameter estimation include timeseries or harmonic analysis data from tide gauges, current measurements (e.g. from ADCPs), or satellite altimetry. Techniques for performing this calibration include Kalman filters (e.g. Mayo et al. (2014)), Bayesian inference approaches (e.g. Hall et al. (2011), Sraj et al. (2014b)), and gradient-based methods utilising adjoint models (e.g. Maßmann (2010)) or other gradient calculation techniques (e.g. Sraj et al. (2014a)). Adjoint-based methods can be efficiently applied to the estimation of very large numbers of control parameters. However, this leads to the problem of overfitting, which is commonly addressed in the literature by either regularisation of the calibration problem (Maßmann, 2010), the reduction of the dimension of the parameter space (Lu and Zhang, 2006, Zhang et al., 2011), truncation of the optimisation after a small number of iterations (Heemink et al., 2002), or combinations of the above. It should be noted that any calibration with respect to the bottom friction parameter will simultaneously be addressing both unknown physical quantities (e.g. related to bottom roughness), as well as errors associated with other model inputs, and modelling and discretisation choices. However, calibration with respect to bottom friction alone is commonly undertaken (e.g. Ullman and Wilson (1998) and several of the above references), and is therefore the focus of this study. The methodology presented can also be applied to calibration with respect to other model inputs.

Any parameter estimation problem requires two components: firstly, a suitable set of observations from which to infer the unknown model parameters, and secondly, an appropriate parameter space within which the unknown 
parameters lie (e.g. which/how many model parameters to estimate, their spatial distribution, etc). These two aspects constitute the 'experiment design', the optimisation of which is known as 'optimal experiment design' (OED), and is the topic of this paper. The optimisation of these two aspects is often considered independently.

The optimisation of the observation strategy amounts to selecting which variables to measure, and when and where to measure them, in order to infer a given set of unknown model parameters. This aspect of OED is common in the literature, with example applications in a variety of fields (Ucinski, 2004). The computation of the model's sensitivities with respect to its unknown inputs is central to the OED method, typically via construction of the Fisher Information Matrix (FIM) (Fedorov, 1972) or something closely related. For a given experiment design, the inverse of the FIM gives a lower bound on the covariance matrix of the estimated parameters (Söderström and Stoica, 1989, Machado et al., 2009, Alaña and Theodoropoulos, 2011). The optimisation of the experiment design can be defined as the minimisation of this parameter covariance, so the crux of FIM-based OED methods is therefore to maximise (a scalar measure of) the FIM. Since the FIM is constructed from model sensitivities, it does not explicitly depend on the values of the model inputs or the observations themselves. The OED process can therefore be performed in advance of expensive observation surveys. FIM-based OED methods have been applied in a variety of experimental sciences (e.g. Balsa-Canto et al. (2008), Strigul et al. (2009), Yu et al. (2018)). Within the field of numerical coastal and estuarine modelling, de Brauwere et al. (2009) utilised an FIM-like method for the calibration of a reactive transport model, designing a sampling strategy to best constrain mortality and sedimentation rates of E. coli in the Scheldt Estuary using a given number of water samples. Vandenberghe et al. (2002) utilised a similar FIM-based method to design an optimal sampling strategy in a water quality model of the River Dender. Graham et al. (2017) take an alternative sensitivity-based approach to optimising experiment design for a storm surge case study, in order to determine an optimal configuration of observation buoys with which to infer a given set of unknown friction parameters. A similar concept to optimal experiment design is that of 'targeted observations' for state estimation (e.g. Langland (2005)). However, while FIM methods have been applied to state estimation (Alaña and Theodoropoulos, 2011), the focus of the present study is on parameter estimation.

The second aspect of experiment design is the selection of the parameters to be estimated. This aspect is the focus of the present study since, as is the case in many similar coastal regions, a reasonable set of observation data already exists within the selected study region, while there is no obvious choice for the input parameter space. A similar and commonly approached problem in the OED literature is the selection of a subset of model parameters for estimation; this has often been solved via FIM methods (Machado et al., 2009, Chu and Hahn, 2009, Kravaris et al., 2013) or other sensitivity analysis-based methods (Li et al., 2004, Wang et al., 2018).

The concept of experiment design for bottom friction parameter estimation has been discussed in numerous studies within the coastal ocean modelling literature. A common approach to designing such experiments is to select a low-dimensional representation of the bottom friction field, so that only a relatively small number of control parameters need to be inferred from the observation data. However, appropriately selecting the control parameters is not a straightforward task. Various methods have been used for the selection of these control parameters (Lardner et al., 1993, Ullman and Wilson, 1998, Chen et al., 2014), with typical schemes involving distributing control points across the model domain either uniformly, based on some physical quantity such as the bathymetry gradient (Lu and Zhang, 2006), or based on the volume and distribution of the observation data (Das and Lardner, 1991, Altaf 
et al., 2012). Other studies have divided their model domain into subdomains based on sedimentology data (Guillou and Thiébot, 2016), land classification (Graham et al., 2017) or bathymetry contours (Sraj et al., 2014b). However, due to the difficult nature of the parameter selection problem, most of the above examples still required some form of regularisation in order to avoid overfitting, despite the modest number of control parameters. This regularisation typically takes the form of a penalty term in the misfit functional, which provides a 'prior' value for the parameter, thus constraining its value where the observations are insufficient to do so. However, while this constitutes a practical approach, one drawback of such regularisation is that the penalty term introduces additional degrees of freedom (e.g. the magnitude of the penalty term, the prior value for the parameter, etc) which must be selected appropriately, e.g. via a cross-validation approach utilising a second observation dataset (Ullman and Wilson, 1998).

Heemink et al. (2002) used an adjoint model to compute the gradient of the model-observation misfit with respect to the spatially varying inputs, in order to inform the selection of a small set of independent control parameters. However, this approach has the disadvantage that the gradient calculation depends on the observation data. As described above, an advantage of OED frameworks is that they are independent of the observation data itself, depending only on the experiment design (i.e. the location and timing of the observations). This enables OED frameworks to be applied in advance of (expensive) observation surveys. This dependence on the observation data also means that the resulting experiment design is implicitly motivated by reducing the model-observation misfit, rather than identifying designs resulting in tight constraints on the control parameters. Furthermore, the approach by Heemink et al. (2002) still did not entirely avoid the overfitting problem, instead relying on truncation of the optimisation algorithm after a small number of iterations. This again requires an additional dataset in order to determine when to terminate the optimisation algorithm, therefore sharing one of the drawbacks of the conventional penalty term regularisation approach.

Within this study, we demonstrate the application of an OED framework to a bottom friction parameter estimation problem. We seek a low-dimensional piecewise-constant representation of the spatially varying bottom friction coefficient within a model of the Bristol Channel, in which all selected control parameters can be constrained by the observations, without the need for regularisation. We proceed via an FIM-based method which utilises an adjointcapable shallow water solver to compute model sensitivities. We use a modified version of the so-called D-criterion as a scalar measure of the FIM. The maximisation of this criterion corresponds to the minimisation of the parameter estimate confidence region, and hence to the experiment design providing the tightest constraints on the unknown parameters. In this respect, the optimal experiment designs identified within this study are motivated by parameter identifiability, and our methodology is therefore distinct from one which is motivated only by minimising modelobservation discrepancy, where parameter uncertainty (and consequently model outputs at uncalibrated locations) may remain high. Note also that this approach can be considered as an alternative to the traditional regularisation approach to avoiding under-constrained problems, since it is possible to obtain a set of control parameters which are constrained by the observations (to a given precision), without the need for regularisation or optimisation truncation as described above. To the authors' knowledge, this paper is the first application of an OED procedure to a coastal ocean parameter estimation problem. Our approach is also novel in its use of the adjoint model for the construction of the FIM, for the purpose of selecting a low-dimensional parameterisation of a fully spatially-varying model input.

The adjoint-capable model used within this work, Thetis, is described in section 2 along with the case study 
region and the observation data used. The optimal experiment design problem is described in section 3 , including detail of the FIM-based optimisation method we employ. Results from the OED framework are presented in section 4 , including verification that our modified D-criterion provides a reasonably tight bound on the size of the true parameter confidence region. This section also explores the influence of the quantity and precision of the observations on experiment design performance. The results from model calibration using our optimal design are summarised in section 5. In section 6, we further demonstrate the OED framework via an application to a model of the northwest European continental shelf. The implications of our results are discussed in section 7 , including avenues for future work. Finally, a summary and conclusions can be found in section 8 .

\section{Model and study region}

Within this work, we take the Bristol Channel and Severn Estuary as a case study region. The Bristol Channel is a macrotidal inlet situated toward the south-east of the UK, and is of significant interest for its tidal range energy resource (Angeloudis and Falconer, 2017). It is also the site of the Hinkley Point nuclear power station. Furthermore, accurate models are vital for flood risk studies (e.g. Lyddon et al. (2018)). As such, the Bristol Channel is a site of particular interest for the development of accurate numerical models.

\subsection{Two-dimensional adjoint-capable shallow water model}

Thetis is a 3D (Kärnä et al., 2018, Pan et al., 2020) and 2D (Vouriot et al., 2019) finite element based coastal ocean model, built using the finite element code generation framework Firedrake (Rathgeber et al., 2016). Within this work, we use Thetis in its two-dimensional depth-averaged mode, solving the nonlinear shallow water equations given by

$$
\begin{aligned}
\frac{\partial \eta}{\partial t}+\nabla \cdot(H \mathbf{u}) & =0 \\
\frac{\partial \mathbf{u}}{\partial t}+\mathbf{u} \cdot \nabla \mathbf{u}+\mathbf{F}_{\mathbf{C}}+g \nabla \eta & =-\frac{\boldsymbol{\tau}_{b}}{\rho H}+\nabla \cdot\left(\nu\left(\nabla \mathbf{u}+\nabla \mathbf{u}^{T}\right)\right),
\end{aligned}
$$

where $\eta$ is the surface elevation, $\mathbf{u}$ is the depth-averaged velocity vector, $H=\eta+h$ is the total water depth, $h$ is the bathymetry (measured positive downwards), $\mathbf{F}_{\mathbf{C}}$ is the Coriolis force, $g$ is the acceleration due to gravity, $\rho$ is the density, $\nu$ is the viscosity, and $\boldsymbol{\tau}_{b}$ is the bottom stress. Within this work, the bottom stress is parameterised via Manning's $n$ formulation

$$
\frac{\tau_{b}}{\rho}=\frac{g n^{2}}{H^{\frac{1}{3}}}|\mathbf{u}| \mathbf{u},
$$

where $n$ is the (spatially varying) Manning coefficient. This coefficient is assigned a uniform value of $n=0.025$ $\mathrm{s} \mathrm{m}^{-1 / 3}$ outside the Bristol Channel, while its value inside the Channel is to be inferred via parameter estimation methods; see figure 1. Wetting and drying of intertidal regions is handled via the bathymetry modification scheme of Kärnä et al. (2011). This scheme introduces a wetting-drying parameter $\alpha$, which controls the transition from wet to dry regions of the domain. Smaller values of $\alpha$ produce more accurate results, but there exists a minimum stable value which depends on the bathymetry gradient and mesh resolution at the wet-dry interface. In this work, $\alpha$ is taken as $0.2 \mathrm{~m}$, which was found by experimentation to be close to the minimum stable value for this model setup.

The unstructured mesh used throughout this study is shown in figure 1, and is based on that used in Mackie et al. (2020b). The mesh was generated on a UTM30 coordinate projection, using the Python package qmesh (version 
1.0.2) (Avdis et al., 2018), which interfaces the mesh generator Gmsh (version 3.0.4) (Geuzaine and Remacle, 2009). The coastline data is from the Global Self-consistent, Hierarchical, High-resolution Geography Database (GSHHG) (Wessel and Smith, 1996). The mesh resolution varies between $250 \mathrm{~m}$ at the innermost part of the Channel, and $8 \mathrm{~km}$ at the ocean boundaries, with an intermediate resolution along other parts of the coastline. This results in a total of 42,862 triangular elements. The bathymetry is taken from Digimap (Digimap, 2013), which has a resolution of approximately $180 \mathrm{~m}$. The ocean boundaries are forced with tidal elevations generated based on the dominant constituents (M2 and S2) from the TPXO database (Egbert and Erofeeva, 2002). The next most significant constituent (N2) has less than half the amplitude of the these primary constituents. While the choice to force with only two constituents neglects any transfer of energy from other tidal constituents, we assume that this forcing is sufficient for the purposes of this study, which represents a proof of concept for our OED framework. A no-normal-flow boundary condition is applied on the coastal boundaries, and river outflow is neglected.

The governing equations (1) are solved using the $\mathrm{P}_{1}^{\mathrm{DG}}-\mathrm{P}_{1}^{\mathrm{DG}}$ finite element pair, with a Crank-Nicolson timestepping method and a timestep size of $\Delta t=200 \mathrm{~s}$. All model runs within this work follow a generic spin-up period of 10 days with a default uniform friction parameter of $n=0.025 \mathrm{~s} \mathrm{~m}^{-1 / 3}$. Following this initial spin-up, each friction configuration is run for a further 17.77 days, with harmonic analysis (for the M2 and S2 constituents) performed on the final 14.77 days. This period was chosen to meet the Rayleigh criterion for resolving the M2 and S2 constituents.

The adjoint mode of Thetis, which is used within this work for the computation of model sensitivity with respect to the Manning coefficient $n$, is generated via pyadjoint (Mitusch et al., 2019, Farrell et al., 2013), which interfaces with Firedrake to efficiently derive the adjoint model. For more detail on Thetis adjoint and its applications, see previous studies e.g. Warder et al. (2019, 2021), Goss et al. (2020).

\subsection{Observation data}

The observations used within this study are the M2 and S2 harmonic amplitudes and phases at the 20 tide gauge locations indicated in figure 1 (National Oceanography Centre, personal communication 2018). This amounts to a total of 80 pieces of data, which can be compared with model outputs. While more harmonic constituents are available within the dataset, the M2 and S2 constituents dominate the dynamics within the Bristol Channel with typical amplitudes of around $4 \mathrm{~m}$ and $1.5 \mathrm{~m}$ respectively, compared with around $0.6 \mathrm{~m}$ for the next most significant constituent (N2). The selected OED methodology incurs a computational cost proportional to the volume of data used for the parameter estimation, so we choose not to consider additional constituents and focus only on the M2 and S2 harmonics. The effect of the volume of observation data (including the choice of constituents) on experiment design performance is considered in section 4.3.

Throughout this work, we assume that some uncertainty is introduced by the observations. Specifically, we assume that the amplitude observations are independent and identically distributed variables, and similarly for the phases, with variances of $25 \mathrm{~cm}^{2}$ and $6.25^{\circ}$, respectively. While this is somewhat arbitrary, we discuss the influence of this choice in section 4.4

\section{Optimal experiment design}

We aim to determine an optimal low-dimensional representation of the spatially varying Manning coefficient field. In particular, we seek a piecewise-constant field, such that the coefficient field is specified by a vector of length $m$. 


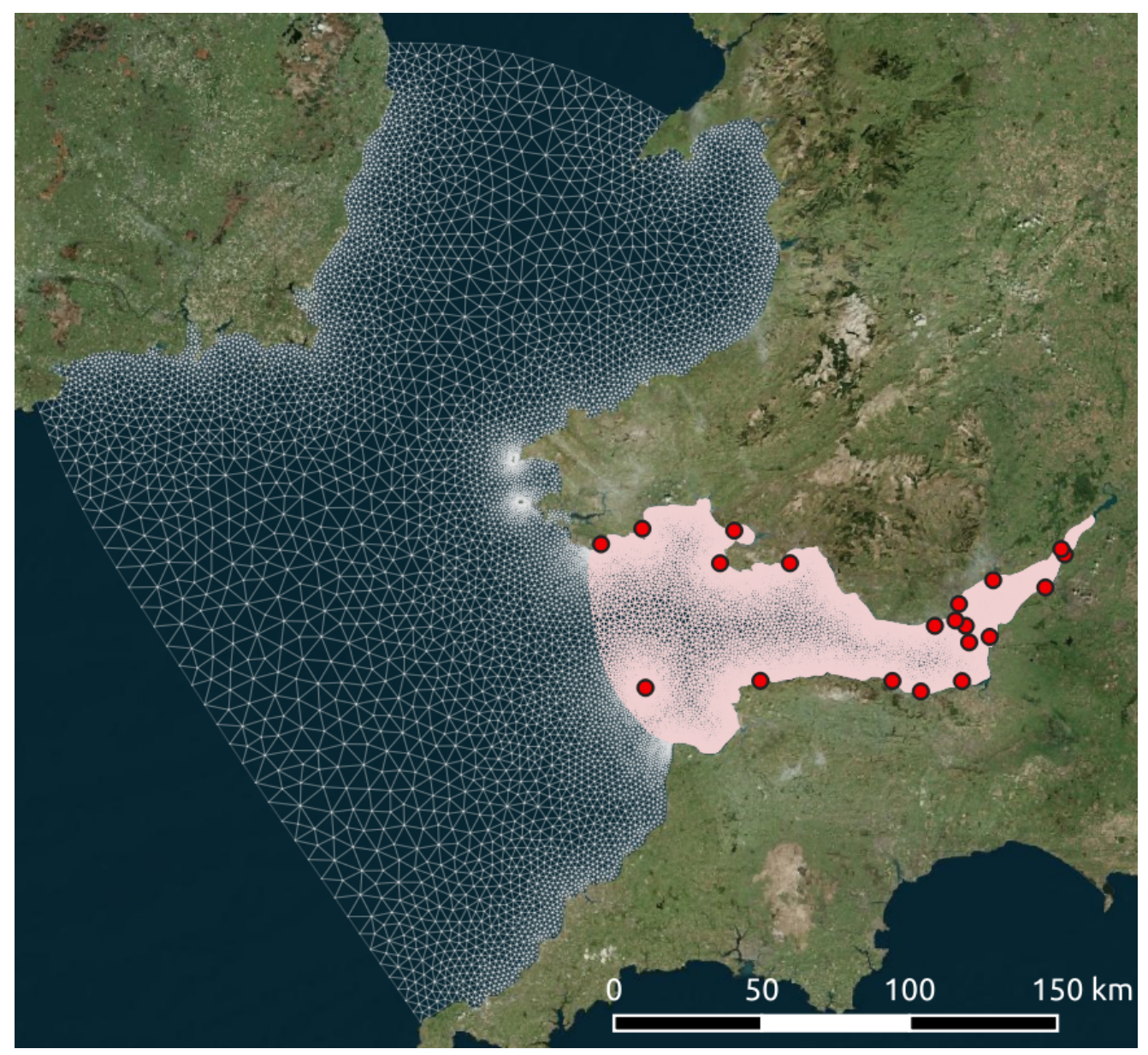

Figure 1: Unstructured mesh used for all simulations within this work. Pink indicates the region where the friction coefficient is permitted to vary spatially. Red circles indicate locations where tidal harmonic data is used.

The aim is to determine the optimal number of parameters $m$, and the optimal mapping from these parameters onto the model domain, such that tight constraints on the parameters can be achieved via a parameter estimation exercise using the observations described in section 2.2. The choice of this mapping and number of parameters will be referred to as the experiment design. We emphasise that this problem is distinct from conventional applications of OED to parameter selection, where an experiment design typically consists of a selection of a subset of parameters, with unselected parameters held constant (e.g. Li et al. (2004), Chu and Hahn (2007), Machado et al. (2009)).

We first apply an a priori constraint on the friction coefficient, by splitting the model domain into subdomains, within each of which the friction coefficient is assumed to be uniform. This constraint permits the use of an exhaustive search algorithm to find the optimal design. The selected number of these subdomains must be sufficiently large that the space of experiment designs contains a 'good' design, but small enough to facilitate the use of the exhaustive search algorithm. Within this work, we consider two such a priori constraints, which are described in section 3.1.

Once a suitable subdomain parameterisation of the friction coefficient has been selected, we explore the remaining space of experiment designs to determine the optimal design. Each design corresponds to a grouping of the a priori subdomains into $m$ groups, such that the friction coefficient is specified by $m$ parameters. In order to rank the experiment designs, we compute the Fisher Information Matrix corresponding to each design, and find the design 
which optimises a selected scalar measure of this matrix. This approach is described in detail in section 3.2.

\subsection{A priori subdomain parameterisations}

Within this work, we consider two different choices for the a priori subdomain parameterisation of the friction coefficient field:

1. Division of the domain by slices drawn by hand, as shown in figure 2. This division is somewhat arbitrary; the number of subdomains was chosen to be the same as the number of sediment classification types present in the domain (eight, see below), with the slices chosen to divide the Bristol Channel fairly evenly, with slightly smaller sections further into the estuary where the density of observation locations is higher. The possible experiment designs are further constrained such that the slices are grouped into contiguous blocks (i.e. region 1 and region 3 cannot be assigned the same friction parameter unless region 2 is also included).

2. Division of the domain based on sediment data, as shown in figure 3. The sediment data is taken from the SHOM database (Service Hydrographique et Océanographique de la Marine) (SHOM, 2013), which divides the domain into discrete sediment types. Since the presence of sediment influences the physical process of momentum loss due to bottom friction, this is an attempt to incorporate physical knowledge into the experiment design. The sediment types within the model domain are shown in figure 3. The candidate experiment designs are further constrained by requiring that the sediment-based subdomains are grouped contiguously in order of sediment grain size; this order is shown in table 1 , along with the area occupied by each sediment type within the model domain.

Each possible experiment design corresponds to a grouping of the selected subdomains, according to the $a$ priori constraints described above. Given that there are eight subdomains, for a parameterisation with $m=2$ (i.e. a grouping of the subdomains into two groups), there are seven possible designs for each choice of a priori constraint. For $m=3$, there are 21 possible designs, and for $m=4$ there are 35 .

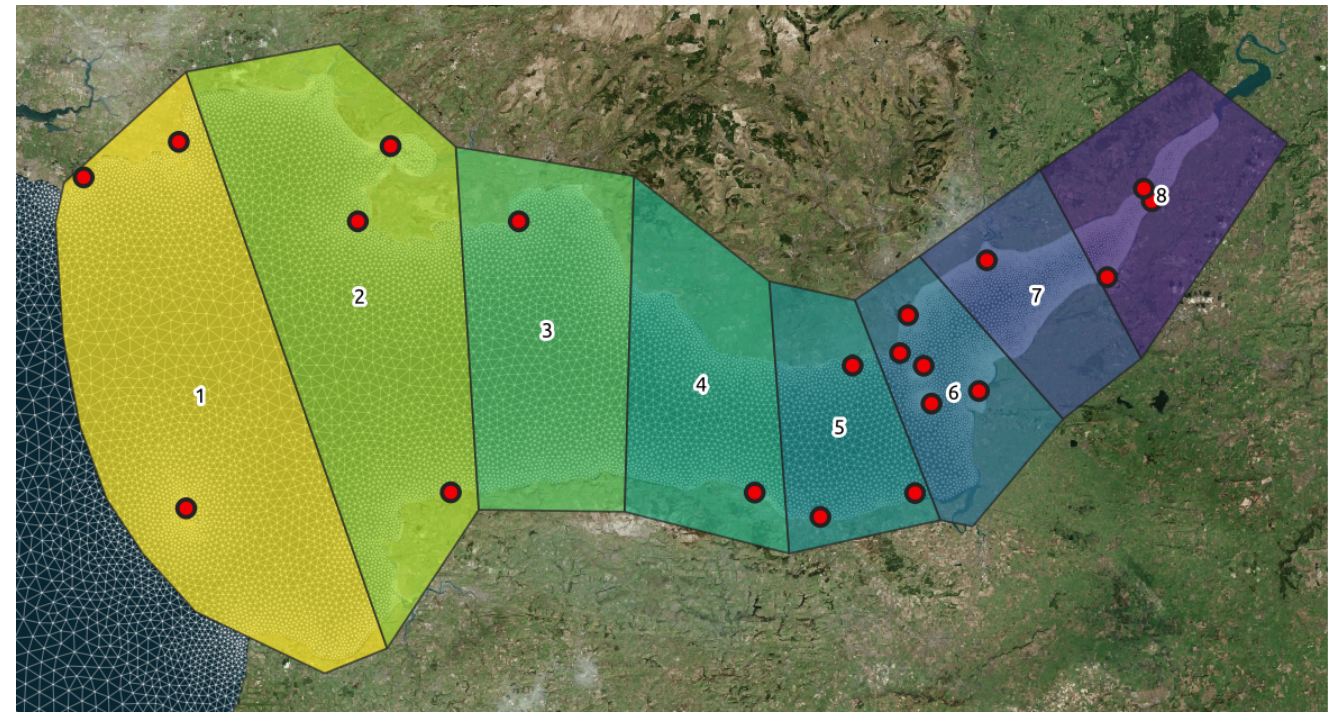

Figure 2: Division of the Bristol Channel into slices, labelled by ID number. Red circles indicate locations where tidal harmonic data is used. 


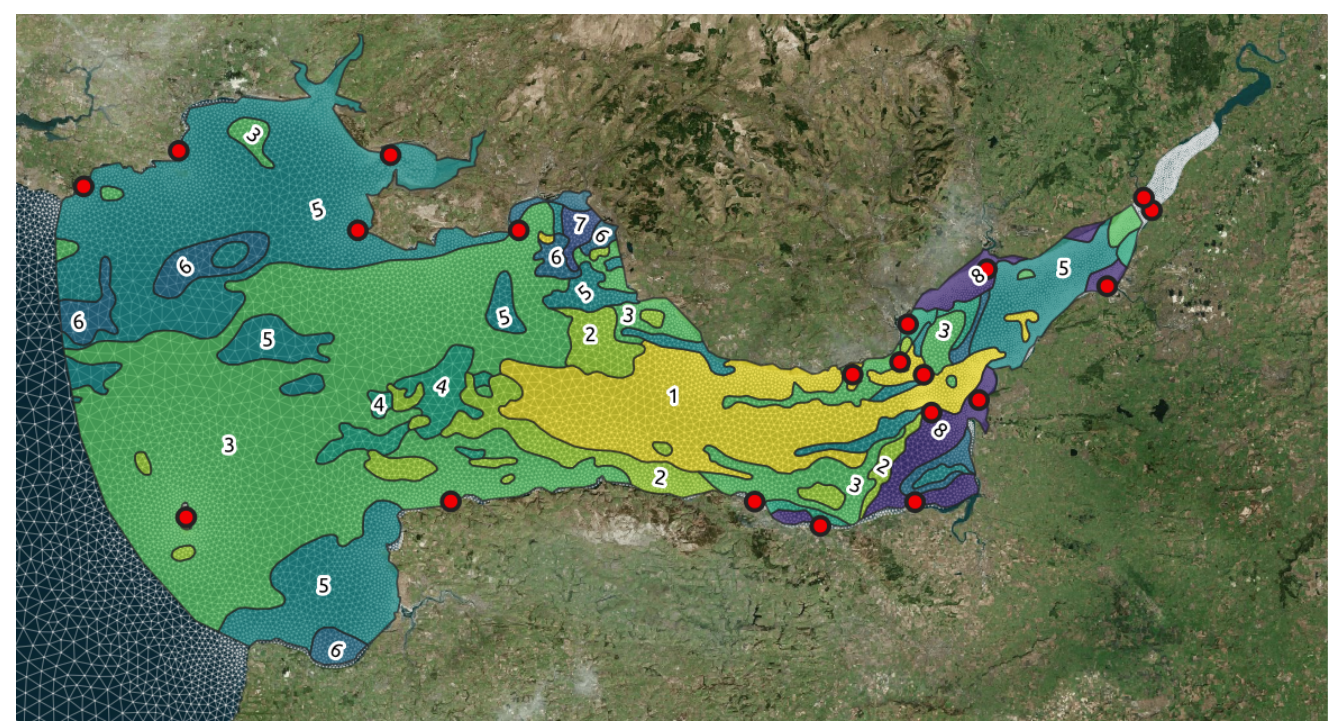

Figure 3: Spatial distribution of sediment types within the Bristol Channel, labelled by ID number. Red circles indicate locations where tidal harmonic data is used.

\begin{tabular}{c|c|c|c|c|c|c|c|c} 
Sediment name & NFRoche & NFG & NFSG & NFSGV & NFS & NFSV & NFVS & NFV \\
\hline ID & 1 & 2 & 3 & 4 & 5 & 6 & 7 & 8 \\
\hline Area covered $/ \mathrm{km}^{2}$ & 761 & 375 & 2800 & 199 & 2051 & 297 & 28 & 216
\end{tabular}

Table 1: Sediment types found within the Bristol Channel (from SHOM dataset), sorted by roughness length.

\subsection{The Fisher Information Matrix}

For reviews of parameter selection methods via the Fisher Information Matrix (FIM), the reader is referred to works from other fields, e.g. Machado et al. (2009), Kravaris et al. (2013). However, note that the problem considered here is subtly different to the conventional parameter selection problem, where the objective is typically to select an optimal subset from a large set of parameters, with unselected parameters taking prescribed values. In this work, we seek an experiment design in which the friction parameter is specified everywhere within the Bristol Channel; each experiment design corresponds to a grouping of model subdomains, not a subset selection. This is perhaps more similar to the clustering of parameters considered by Chu and Hahn (2009), although we take a different approach. The following is a simple exposition of the FIM approach to optimal experiment design.

Consider a parameter estimation problem, where a vector of unknown parameters $\boldsymbol{\theta}$ is to be estimated by minimising a misfit functional given by

$$
(\mathbf{y}-\mathbf{f}(\boldsymbol{\theta}))^{T} \operatorname{cov}_{e}^{-1}(\mathbf{y}-\mathbf{f}(\boldsymbol{\theta}))
$$

with respect to $\boldsymbol{\theta}$, where $\mathbf{y}$ and $\mathbf{f}(\boldsymbol{\theta})$ are vectors of observed and modelled quantities respectively, and $\operatorname{cov}_{e}$ is the observation error covariance matrix. The parameter covariance matrix resulting from the minimisation of equation (3) can be estimated as

$$
\operatorname{cov}_{p}=\left(J^{T} \operatorname{cov}_{e}^{-1} J\right)^{-1},
$$


where $J$ is the Jacobian matrix with elements given by

$$
J_{i j}=\frac{\partial f_{i}(\boldsymbol{\theta})}{\partial \theta_{j}} .
$$

It is common within the literature to utilise a forward difference method to compute this Jacobian matrix. In this work we instead use the adjoint mode of the numerical model; this is described in section 3.2.3.

The parameter covariance given by equation (4) does not depend on the observations $\mathbf{y}$, but only on their error covariance. Note, however, that it implicitly depends on the number, location and timing of the observations, and other aspects of the experiment design. For a given experiment design, the parameter covariance can therefore be estimated based only on the model sensitivities and the observation covariance, and hence can be estimated prior to solving the parameter estimation problem, and without committing resources to observation surveys or model inversion.

Within the optimal experiment design literature, it is common to work with the inverse of the parameter covariance matrix, the so-called Fisher Information Matrix (FIM) (Fedorov, 1972), defined as

$$
\mathrm{FIM}=J^{T} \operatorname{cov}_{e}^{-1} J .
$$

Optimisation of the experiment design is undertaken by finding the configuration (in general, the timing and location of observations, or selection of the input parameter space) which optimises some scalar measure of the FIM. There are a variety of common choices for this scalar measure in the literature (Emery and Nenarokomov, 1998, Kravaris et al., 2013).

Within this work, we use a modified version of the so-called D-criterion, which we motivate here. In the case of estimating a single parameter, the optimal experiment design can be defined as the one which minimises the confidence interval of the estimated parameter. The higher-dimensional analogue of this is that the optimal design minimises the volume of the confidence ellipsoid of the estimated parameters. This volume is characterised by the determinant of the parameter covariance matrix, $\operatorname{det}\left(\operatorname{cov}_{p}\right)$ (Walter and Pronzato, 1990). Since the FIM is the inverse of $\operatorname{cov}_{p}$, the minimisation of this confidence ellipsoid volume corresponds to the maximisation of $\operatorname{det}(\mathrm{FIM})$. The so-called D-criterion is simply defined as this determinant, $\operatorname{det}(\mathrm{FIM})$.

Within this work, we define a modified D-criterion given by

$$
\operatorname{ModD}=\operatorname{det}(\mathrm{FIM})^{1 / m},
$$

where $m$ is the number of parameters to be estimated. This is sometimes given as the standard definition of the Dcriterion (e.g. Emery and Nenarokomov (1998)), but for clarity we refer to this as the modified D-criterion, or ModD. In terms of parameter confidence ellipsoids, the maximisation of the ModD criterion corresponds to the minimisation of the geometric mean of the principal dimensions of the parameter confidence ellipsoid, whereas the standard Dcriterion corresponds to minimising the parameter confidence ellipsoid volume. For a fixed $m$, the experiment design which maximises ModD also maximises the standard D-criterion. However, the inverse power of $m$ ensures that the dimensions of the ModD criterion are independent of the number of parameters estimated, so that its value can be directly compared between experiment designs with different numbers of parameters. 


\subsubsection{A note on nonlinearity}

The above exposition is only strictly valid when the model is linear. If the model is nonlinear with respect to the unknown parameters (as is the case within this work, since the numerical tidal model is nonlinear), equation (4) is not exact, but the right-hand-side is nevertheless a measure of the maximum achievable parameter precision (de Brauwere et al., 2009). The inverse of the FIM (as defined by equation (6)) is considered to give a lower bound on the parameter error covariance matrix (Alaña and Theodoropoulos, 2011). That is,

$$
\operatorname{cov}_{p} \geq \mathrm{FIM}^{-1},
$$

where the inequality is understood to mean that $\left(\operatorname{cov}_{p}-\mathrm{FIM}^{-1}\right)$ is positive semidefinite (Söderström and Stoica, 1989, Emery and Nenarokomov, 1998, Machado et al., 2009).

Nonlinearity also means that the Jacobian, and therefore the FIM, are functions of the unknown parameters. The optimisation of the modified D-criterion as defined above therefore identifies only a 'locally optimal' experiment design (Ford et al., 1989, Huan and Marzouk, 2013). That is, the optimal experiment design is optimal only within a region of the parameter space local to the initial parameter estimate, where the model response is approximately linear. This issue can be overcome with sequential or iterative design (Blanchet et al., 2008, Catania and Paladino, 2009), or maximin optimisation (Pepelyshev et al., 2004, Rojas et al., 2007, Sun, 2007, Ushijima and Yeh, 2015), but such designs are outside the scope of this study. Instead, it is assumed that an initial estimate for the friction coefficient of $n=0.025 \mathrm{~s} \mathrm{~m}^{-1 / 3}$ is appropriate, and that the model response to perturbations in the friction coefficient from this value can be assumed to be linear. The results presented in section 4.1 suggest that this is an acceptable assumption, and that the bound provided by equation (8) is reasonably tight.

\subsubsection{Defining a 'good' experiment design}

It is useful to consider the values of the ModD criterion for which the corresponding experiment design produces acceptable constraints on the estimated parameters. This is particularly important when comparing experiment designs with different numbers of unknown parameters. In the context of estimating Manning coefficients, which typically vary between around 0.01 and $0.04 \mathrm{~s} \mathrm{~m}^{-1 / 3}$ depending on bed composition (Arcement and Schneider, 1989), a 'good' parameter estimate variance might be $10^{-6} \mathrm{~s}^{2} \mathrm{~m}^{-2 / 3}$. Extending this to higher dimensions analogously to the definition of the ModD criterion, we therefore seek designs which satisfy

$$
\operatorname{det}\left(\operatorname{cov}_{p}\right)^{1 / m}<10^{-6} \mathrm{~s}^{2} \mathrm{~m}^{-2 / 3}
$$

and therefore

$$
\operatorname{det}\left(\operatorname{cov}_{p}\right)^{-1 / m}>10^{6} \mathrm{~m}^{2 / 3} \mathrm{~s}^{-2} .
$$

Since the FIM provides a bound on $\operatorname{cov}_{p}$ via equation (8), we therefore seek designs satisfying

$$
\operatorname{det}(\mathrm{FIM})^{1 / m}>10^{6} \mathrm{~m}^{2 / 3} \mathrm{~s}^{-2},
$$

or equivalently

$$
\operatorname{ModD}>10^{6} \mathrm{~m}^{2 / 3} \mathrm{~s}^{-2} .
$$

Due to the inequality in equation (8), an experiment design satisfying equation (10) is not guaranteed to achieve the target parameter estimate precision given by equation (9). However, we show in section 4.1 that the bound 
provided by the FIM on $\operatorname{cov}_{p}$ appears to be reasonably tight, and therefore that equation (10) is a good indicator of experiment designs satisfying equation (9). We emphasise that the value of the OED method is that ModD can be computed in advance of the parameter estimation procedure, and therefore equation (10) provides a useful a priori indicator of experiment design performance.

\subsubsection{Construction of the Jacobian via the adjoint model}

The crux of computing the FIM is the construction of the Jacobian (equation (5)), which consists of the sensitivity of each model output with respect to each unknown parameter. For this application, the model outputs are the M2 and S2 harmonic amplitudes and phases at 20 locations within the domain, and the model inputs correspond to the set of friction parameters, denoted $\boldsymbol{\theta}$. The difficulty in computing these sensitivities for an arbitrary experiment design arises from the fact that the mapping from the friction parameters onto the model domain is unique to each experiment design. Here, we use the numerical adjoint model to overcome this issue.

An arbitrary spatially varying Manning coefficient field can be represented by a vector $\mathbf{n}$ of length $N$, with the elements of the vector corresponding to the value of the Manning coefficient at each mesh node, and $N$ the number of mesh nodes within the region of variable friction. For a given experiment design, denoted $\mathcal{D}$, we have

$$
\mathbf{n}=\mathcal{D} \cdot \boldsymbol{\theta}
$$

where $\boldsymbol{\theta}$ is the parameter vector of length $m$. The experiment design $\mathcal{D}$ is an $N \times m$ matrix consisting of ones and zeros, which maps the parameters $\boldsymbol{\theta}$ onto the mesh nodes.

First, we use the adjoint model to compute the Jacobian with respect to $\mathbf{n}$, given by

$$
\frac{\partial f_{i}}{\partial n_{j}}
$$

This requires one adjoint model run for each observation $i$, i.e. for each row of the above matrix. There are 80 observations (M2 and S2 amplitude and phase at 20 locations), so the computation of this Jacobian requires 80 adjoint runs. The Jacobian matrix with respect to the parameter vector $\boldsymbol{\theta}$ can then be computed as

$$
\begin{aligned}
J_{i j} & =\frac{\partial f_{i}}{\partial \theta_{j}} \\
& =\frac{\partial f_{i}}{\partial n_{k}} \cdot \frac{\partial n_{k}}{\partial \theta_{j}} \\
& =\frac{\partial f_{i}}{\partial n_{k}} \cdot \mathcal{D}_{k j},
\end{aligned}
$$

where the $\frac{\partial f_{i}}{\partial n_{k}}$ term has been computed via the set of adjoint model runs as described above.

Note that, for a given model setup (mesh, etc), the adjoint model runs are a one-off computational overhead, and the Jacobian with respect to the parameter vector can be computed via equation (13) at negligible additional computational cost, for an arbitrary design $\mathcal{D}$. The computational cost of each adjoint model run is on the same order of magnitude as that of a forward model run. Note that the common approach in the literature to construct Jacobians for OED is to use forward differences, based on running the forward model with perturbed values for each model input. While such an approach would be feasible within this study due the relatively strong a priori constraints described in section 3.1, it would rapidly become infeasible as a greater space of possible experiment designs is considered. In the most general case, the forward differences approach would require $N+1$ forward model 
runs, where $N$ is $\mathcal{O}(10,000)$ for the mesh used in this work; this is clearly not computationally feasible. The use of an adjoint model is a prerequisite for the extension of this OED method to more complex experiment design spaces, and we therefore take the adjoint approach here. To the authors' knowledge this is the first OED study to apply adjoint methods for this purpose.

\section{OED Results}

In section 4.1, we verify that the ModD criterion, computed via the FIM, provides a reliable indicator of experiment design performance. Section 4.2 then summarises the optimal designs returned by the OED framework, as a function of the number of parameters $m$ and the choice of a priori subdomain division. Finally, sections 4.3 and 4.4 address the effects of data quantity and measurement uncertainty on experiment design performance.

\subsection{Verification of the ModD criterion as a measure of design performance}

The effect of the inequality in equation (8), which represents the nonlinearity of the system, is that the FIM approach is expected to overestimate the true performance of a given experiment design. Here, the aim is to investigate the significance of this inequality, i.e. to verify whether the FIM approach is suitable for identifying 'good' experiment designs within the friction parameter estimation context. We proceed by utilising a Bayesian inference framework to solve the parameter estimation problem corresponding to a selection of experiment designs, computing the resulting parameter covariance matrix for each. We can then compare the ModD criterion with the equivalent measure of this parameter covariance matrix, given by

$$
\operatorname{det}\left(\operatorname{cov}_{p}\right)^{-1 / m} .
$$

If the inequality of equation (8) were replaced with an equality, the expression given by equation (14) would exactly equal the ModD criterion. By comparing equation (14) with ModD, we can therefore investigate the significance of the inequality in equation (8) in characterising experiment designs.

While the OED approach described in this paper is applicable to a variety of parameter estimation procedures, here we choose to use a Bayesian inference approach via a Markov Chain Monte Carlo (MCMC) algorithm to solve selected parameter estimation problems, since it is a straightforward and well-studied method which yields a direct estimate of the parameter covariance matrix. Details of the MCMC algorithm, which utilises a Gaussian process emulator as a surrogate for the full numerical model, can be found in Appendix A, and here we simply summarise the results.

We test the experiment designs using both 'synthetic' and 'real' experiments. In the synthetic cases, we use the outputs from the numerical model as observation data within the parameter estimation algorithm. A synthetic result is produced separately for each experiment design, with a friction coefficient field consistent with the design. This

ensures that the optimum model-observation error is exactly zero for each parameter estimation experiment. While in this case the 'observation' uncertainty is also zero, within the Bayesian inference algorithm we specify observation uncertainties as described in section 2.2, in order to generate parameter covariances consistent with our assumptions about the real observation uncertainties. The 'real' experiments use the real-world observations, where we again specify the observation uncertainties as described in section 2.2 . 
For a selection of experiment designs, table 2 presents values of both the ModD criterion and equation (14) resulting from the synthetic and real experiments. We find that, while the $\operatorname{det}\left(\operatorname{cov}_{p}\right)^{-1 / m}$ values are smaller than the ModD values (as expected due to the inequality in equation (8)), they are reasonably close to the bound provided by ModD, and therefore that the ModD value is a good indicator of experiment design performance.

\begin{tabular}{c|c|c|c} 
Experiment design & $\operatorname{ModD}\left[\mathrm{m}^{2 / 3} \mathrm{~s}^{-2}\right]$ & $\begin{array}{c}\operatorname{det}\left(\operatorname{cov}_{p}\right)^{-1 / m}\left[\mathrm{~m}^{2 / 3} \mathrm{~s}^{-2}\right] \\
\text { using synthetic observations }\end{array}$ & $\begin{array}{c}\operatorname{det}\left(\operatorname{cov}_{p}\right)^{-1 / m}\left[\mathrm{~m}^{2 / 3} \mathrm{~s}^{-2}\right] \\
\text { using real observations }\end{array}$ \\
\hline $\begin{array}{c}\text { Slice-based designs } \\
\text { Optimal } m=2 \text { design }\end{array}$ & $5.98 \times 10^{6}$ & $5.63 \times 10^{6}$ & $5.95 \times 10^{6}$ \\
\hline Optimal $m=3$ design & $1.73 \times 10^{6}$ & $1.25 \times 10^{6}$ & $1.62 \times 10^{6}$ \\
\hline Worst $m=3$ design & $0.365 \times 10^{6}$ & $0.357 \times 10^{6}$ & $0.357 \times 10^{6}$ \\
\hline Optimal $m=4$ design & $0.824 \times 10^{6}$ & $0.801 \times 10^{6}$ & $0.794 \times 10^{6}$ \\
\hline $\begin{array}{c}\text { Sediment-based designs } \\
\text { Optimal } m=3 \text { design }\end{array}$ & $0.871 \times 10^{6}$ & $0.691 \times 10^{6}$ & $0.825 \times 10^{6}$
\end{tabular}

Table 2: Comparison of parameter estimation experiment performance using synthetic and real data.

\subsection{Exploration of optimal designs}

Having verified in section 4.1 that the ModD criterion constitutes a good metric of experiment design performance, here we explore the full space of possible experiment designs (for $m=2,3,4$ and 5). Figure 4 shows the ModD criterion values returned by all possible experiment designs, as a function of the number of parameters in the design, $m$, for both choices of a priori constraint as summarised by figures 2 and 3 . The optimal designs for each value of $m$ are shown in figures 5 and 6 for the slice and sediment subdomains, respectively. We make the following observations:

(i) The maximum achievable values of the ModD criterion decrease as the number of unknown parameters $m$ increases. This is expected, since the information provided by the observation data remains constant, but is used to attempt to constrain an increasing number of parameters.

(ii) The designs based on slice subdomains perform better than those based on sediment subdomains. This can be attributed to the fact that several of the sediment types $(2,4,6,7,8)$ correspond to only small regions of the model domain. The performance of a given grouping of sediment types is therefore dominated by the subdomains with the largest area $(1,3,5)$. The space of possible experiment designs is effectively smaller than for the slice-based subdomains, and the maximum achievable ModD criteria are therefore reduced.

(iii) For the slice subdomains, we find that it is possible to achieve the target criterion $\left(10^{6} \mathrm{~m}^{2 / 3} \mathrm{~s}^{-2}\right)$ when grouping the friction subdomains into two or three groups $(m=2$ or 3$)$, but no four-parameter designs meet the threshold ModD criterion.

(iv) For sediment subdomains, we find that it is only possible to meet the threshold ModD criterion using $m=2$, with no possible designs for $m \geq 3$ exceeding the threshold.

(v) In most cases, the ranges of possible ModD criteria for successive values of $m$ overlap. For example, the best possible $m=4$ designs outperform the worst possible $m=3$ designs. This emphasises the value of an 

priori subdomain division. The horizontal dashed line indicates the threshold ModD criterion value as described in section 3.2 .2 .
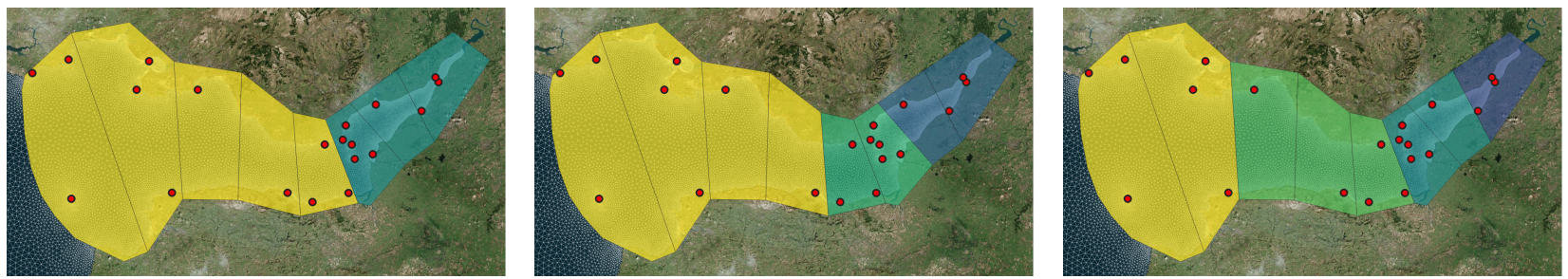

Figure 5: Optimal designs for $m=2$ (left), $m=3$ (centre) and $m=4$ (right), based on the use of slice subdomains.
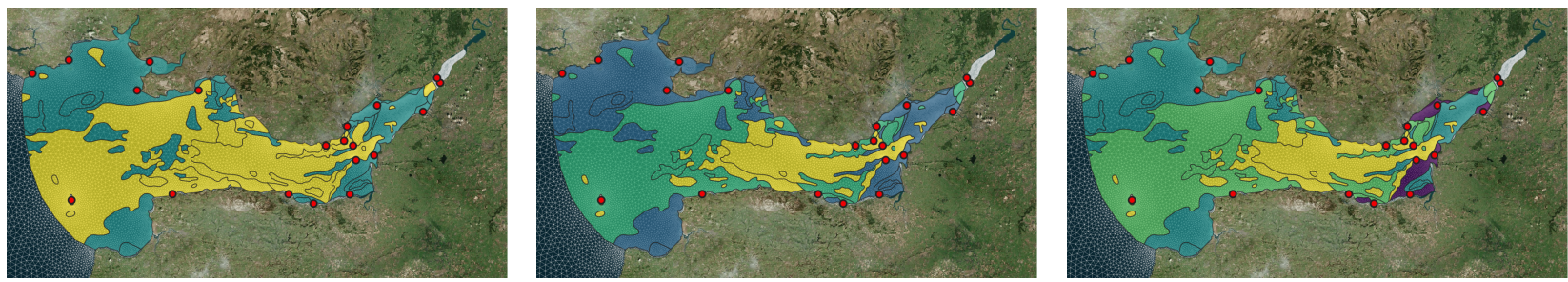

Figure 6: Optimal designs for $m=2$ (left), $m=3$ (centre) and $m=4$ (right), based on the use of sediment subdomains. 


\subsection{Response of OED criterion to data availability}

Here, we investigate the response of the optimal designs, and their corresponding ModD values, to the availability of observation data. To do this, we perform the OED procedure using subsets of the available observations, varying the number of observation locations, and the types of observation data used (i.e. M2/S2 harmonic constituents, amplitude/phase data). This reveals the redundancy in the information provided by the observation dataset, and gives insight into the properties of an experiment design which contribute to its efficacy within parameter estimation.

Figure 7 shows the optimal value of the ModD criterion (on the $y$-axis), and the corresponding optimal experiment design (indicated by symbols as detailed in the legend), as a function of the number of gauges used, for 3-parameter designs based on slice subdomains using various combinations of observation types. Equivalent results from the use of sediment data for the a priori subdomains are shown in figure 8. We make the following observations:

(i) The sensitivity of the ModD criterion to the number of gauges used decreases, and appears to approach zero, as the number of gauges increases. This can be attributed to the fact that the observation datasets contain redundant information, especially since many of the observation locations are clustered together spatially (see figure 1).

(ii) For small numbers of observations, the greatest increase in the ModD value is achieved by adding more gauge locations. For large numbers of observations, the ModD value is most efficiently increased by including different observation types (observations of phases in addition to amplitudes, and/or the inclusion of more harmonic constituents in the analysis). This result has possible implications for observation strategies, since it provides guidance on whether it is most efficient to spread observation infrastructure over a wider area (i.e. add more gauges) or focus on more detailed observations in one location (i.e. observe more constituents).

(iii) For the slice-based subdomains, the optimal design for the maximum number of gauges is the same for all combinations of observation types. Furthermore, only six different optimal designs are found, for any combination of number of gauges and observation types. In other words, the optimal design is relatively insensitive to the volume of data or the types of observations considered. In contrast, the optimal designs are more variable for the sediment-subdomain case. This can be attributed to the fact that many of the sediment types correspond to only very small areas of the Bristol Channel, and subtly different designs therefore have very similar performance, as was shown to be the case in section 4.2 .

As a result of the above observations, we conclude that the a priori use of slice-based subdomains gives cleaner results, which are easier to interpret, than those based on sediment types, as well as producing overall higher values of the ModD criterion and performing better in parameter estimation problems in terms of the achievable parameter constraints. This is largely due to the more even division of the model domain into slices, compared with the sediment-based division, which resulted in several subdomains of small area. Note, however, that this result does not necessarily suggest that the use of sediment data to constrain the spatial distribution of the friction parameter is detrimental to the model performance, or that the sediment data is inconsistent with the observations. 


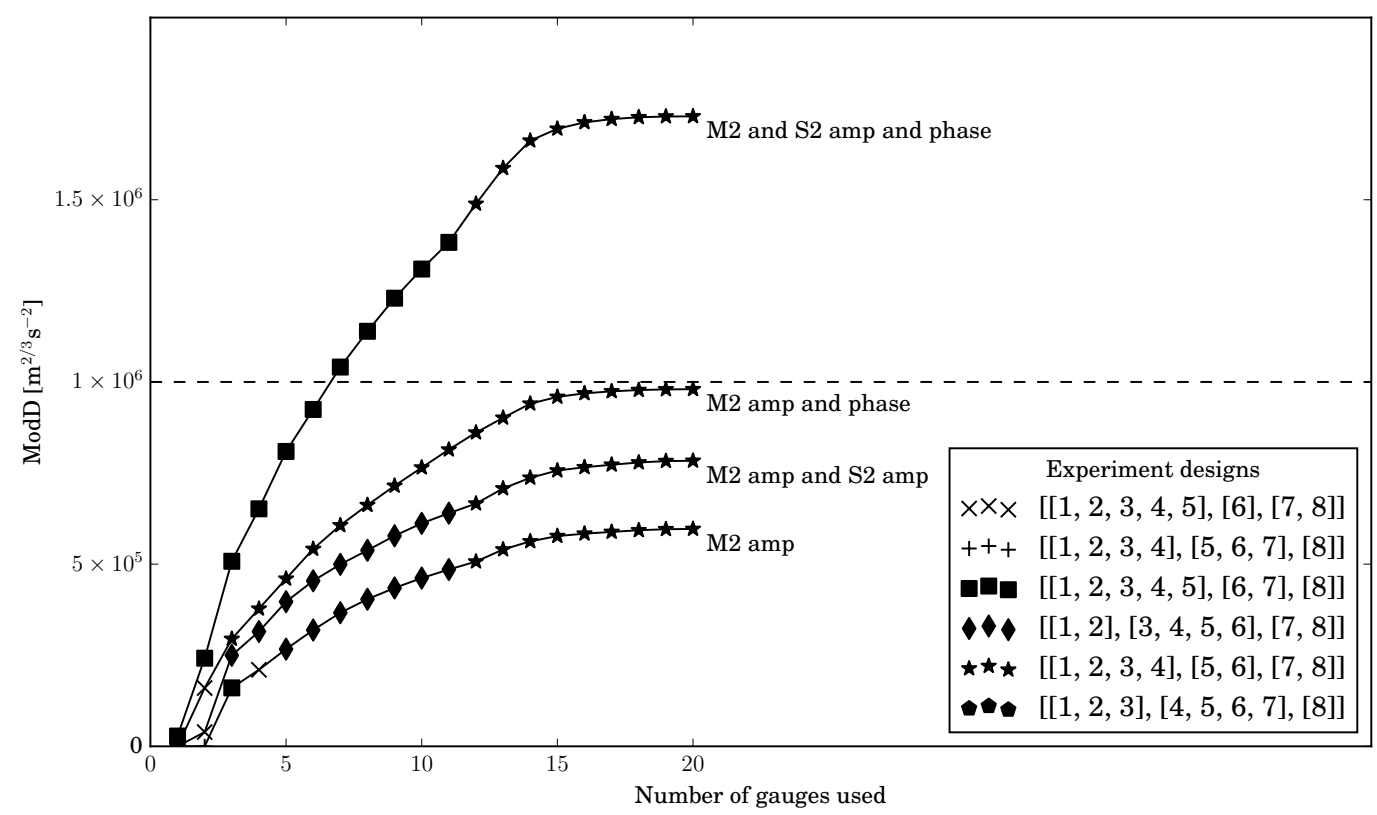

Figure 7: ModD criterion ( $y$-axis) and optimal experiment design (symbols, see legend), as a function of the number of tide gauges used, for four combinations of observation types, based on the use of slice subdomains with $m=3$. As the number of observation locations increases, the achievable ModD criterion increases. Similarly, the use of multiple types of observations (i.e. phases in addition to amplitudes, or the use of data for multiple harmonic constituents) increases the achievable ModD criterion. The optimal experiment design (i.e. the optimal grouping of friction subdomains) is also dependent on the exact set of gauges and observation types used.

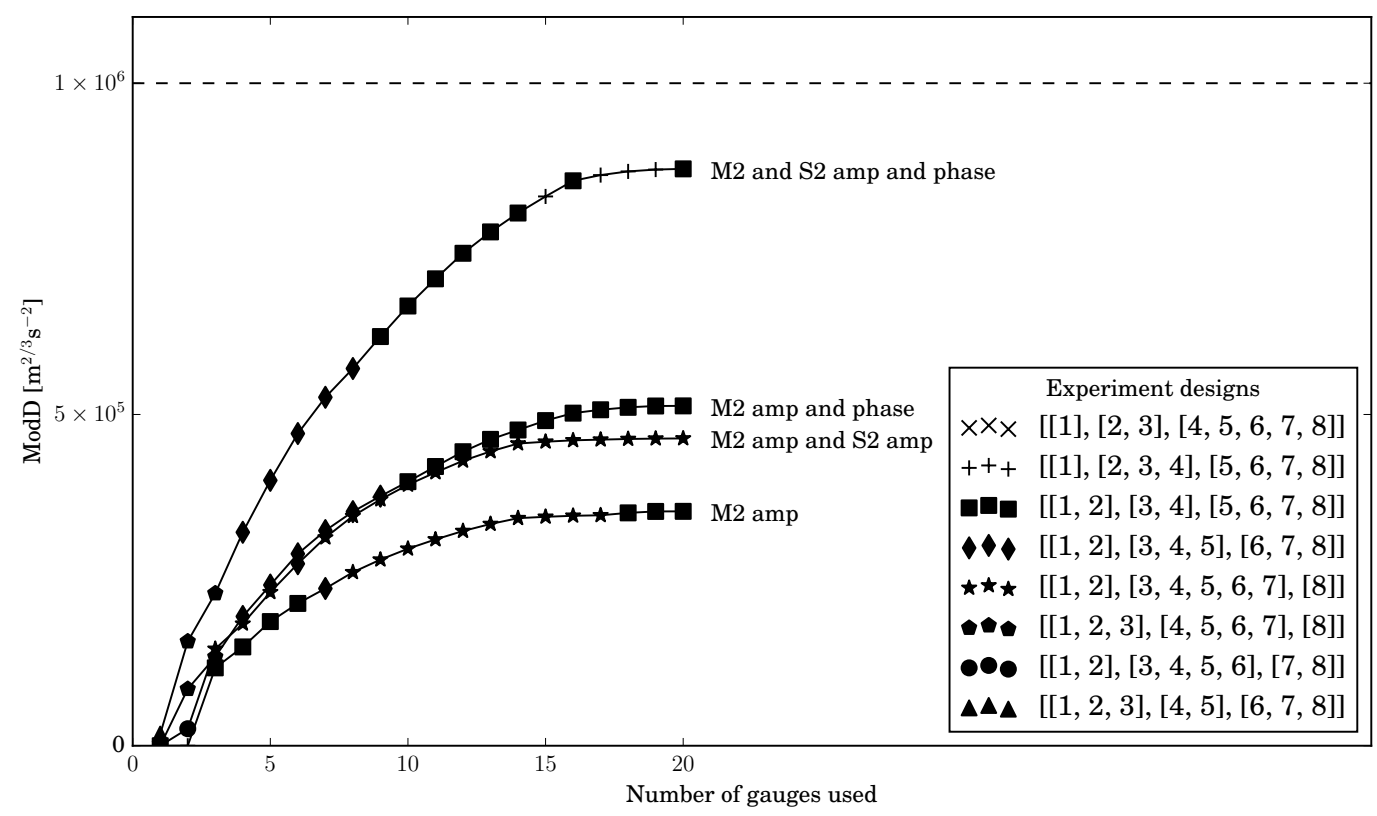

Figure 8: ModD criterion ( $y$-axis) and optimal experiment design (symbols, see legend), as a function of the number of tide gauges used, for four combinations of observation types, based on the use of sediment subdomains with $m=3$. The results show similar features to those of figure 7 . 


\subsection{Response of OED criterion to observation uncertainty}

In this section, we briefly consider the response of the optimal designs to changes in the estimate of $\operatorname{cov}_{e}$. In many optimal experiment design studies, the observation covariance matrix $\operatorname{cov}_{e}$ is considered to be proportional to the identity matrix and is therefore omitted from the formulation of equation (6). However, here we are considering observations of different dimensions (amplitude and phase), and also compare ModD criterion values with a threshold value; the inclusion of $\operatorname{cov}_{e}$ is therefore necessary.

Equation (6) implies that scaling $\operatorname{cov}_{e}$ by a constant simply scales the ModD values inversely. For example, decreasing $\operatorname{cov}_{e}$ by a constant factor would have the effect of translating the data of figure 4 in the positive $y$ direction, meaning that a greater number of experiment designs would meet the ModD threshold value. In other words, observations with smaller uncertainty could be used to achieve a given constraint on a larger number of unknown parameters, or equivalently, fewer observations would be required to achieve a given parameter constraint.

In terms of impact on future observation surveys, it is perhaps useful to note the possible trade-off between low observation uncertainty and the compilation of a diverse set of observations. Based on figure 7, we note that there is a factor of around three between the ModD values for the assimilation of all data (M2 and S2 amplitude and phase), compared with M2 amplitude data only. To achieve the same increase in the ModD criterion using only M2 amplitude data would require a reduction by the same factor in the observation error variance. This demonstrates the balance which can be struck between investing in higher-precision observation techniques and diversifying the observations made. For example, the OED methodology of this study could be used to compare the value of a large number of cheap but high-uncertainty observations with a smaller set of expensive but low-uncertainty observations.

\section{Performance of the calibrated model}

Here we briefly summarise the results from performing the model calibration, using the optimal $m=3$ slice-based experiment design. Figure 9 shows the posterior probability density function (PDF) resulting from the MCMC parameter estimation algorithm, which demonstrates the tight constraints on the control parameters achieved with this experiment design. To construct the 'calibrated' model, we take the mean of this posterior parameter PDF, resulting in the friction coefficient field depicted in figure 10.
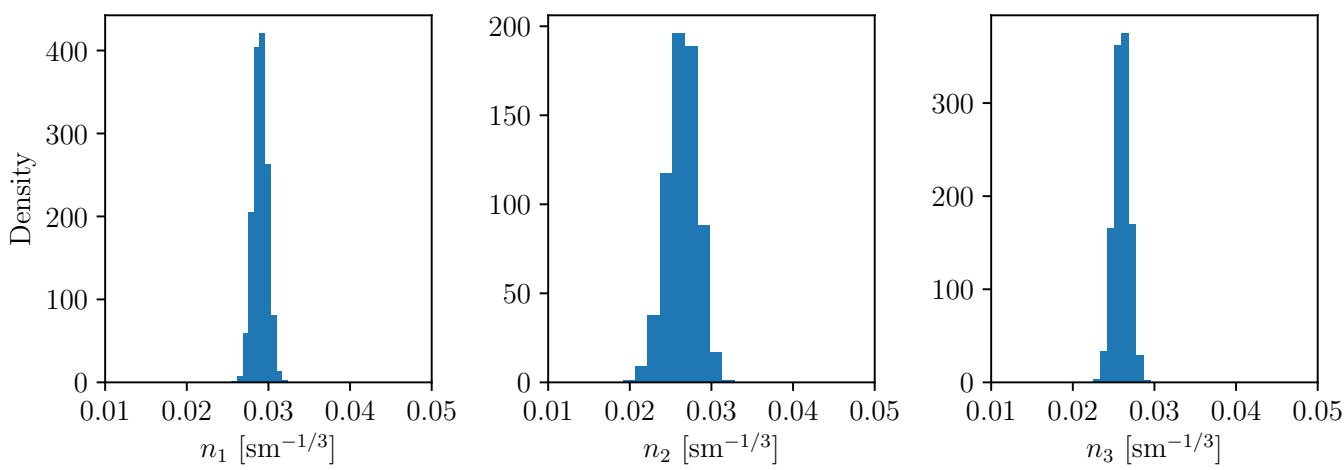

Figure 9: Posterior probability density function for parameter estimation using the optimal $m=3$ slice-based experiment design. 


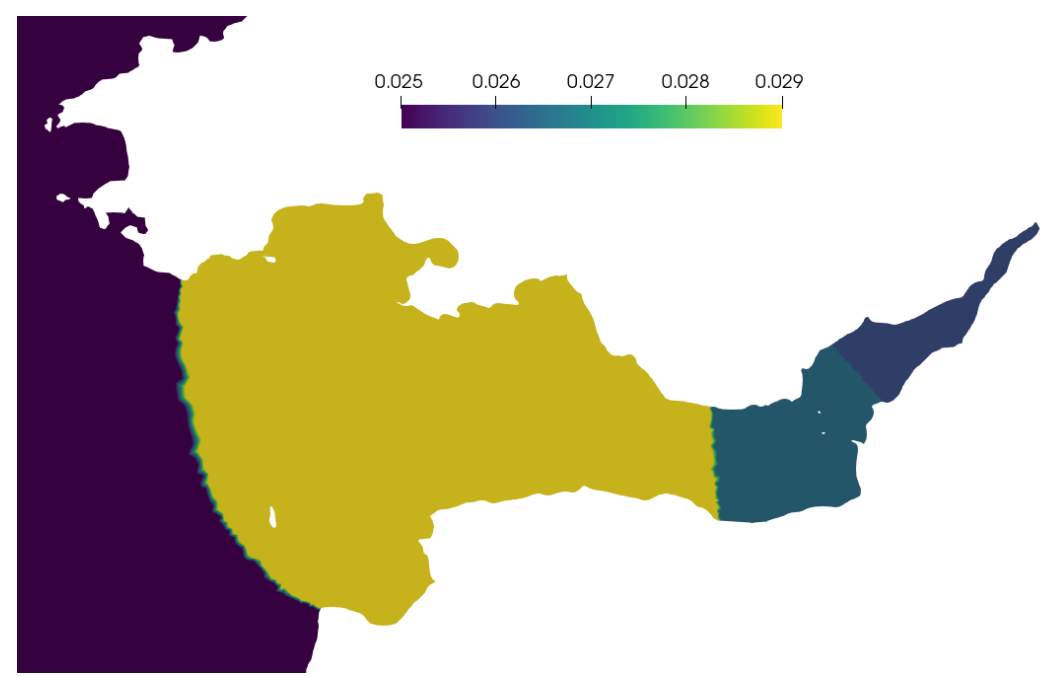

Figure 10: Calibrated Manning coefficient field, using the optimal $m=3$ slice-based experiment design. Units: $\mathrm{s} \mathrm{m}^{-1 / 3}$.

To demonstrate the improvement in performance achieved via this model calibration, we make a comparison with a uniform parameter with with $n=0.025 \mathrm{~s} \mathrm{~m}^{-1 / 3}$. This comparison is shown in figure 11 . The reduction in scatter between the modelled and observed values is easily visible by eye, and is also evident in the root mean squared errors, which reduce from $9.7 \mathrm{~cm}$ and $4.1^{\circ}$ before calibration, to $5.6 \mathrm{~cm}$ and $2.7^{\circ}$ after calibration. We emphasise that further calibration with a larger number of control parameters would enable further reduction in these errors. However, the OED framework tells us that increasing the number of control parameters is not justified, given the observation data used to constrain the unknown parameters.

\section{Application of OED framework to a model of the northwest European continental shelf}

In this section, we present the application of the OED framework to another model domain in order to further demonstrate the power of the method. We choose to use a model of the northwest European continental shelf. The model mesh is shown in figure 12a, which also indicates the locations of 42 tide gauges where observation data is available. We force the model at its ocean boundaries with the M2, S2, K1 and O1 tidal constituents. Each model run spans approximately 50 days, with the final 14.77 days used for harmonic analysis of model outputs. As described in section 3 , the OED framework proceeds as follows:

1. Define the observation dataset. We use the M2 and S2 amplitude and phase observations at each of the 42 tide gauges indicated in figure $12 \mathrm{~b}$, resulting in a total of 168 data points.

2. Select a model setup with some suitable initial estimate for the Manning coefficient (we use a uniform value of $n=0.025 \mathrm{~s} \mathrm{~m}^{-1 / 3}$ ). Using the adjoint model, compute the gradient of each model output (corresponding to the 168 observations) with respect to the fully spatially varying bottom friction parameter. This requires a total of 168 adjoint model runs.

3. Propose a decomposition of the model domain into small regions, within which the bottom friction coefficient will always be uniform. Here we divide our model domain into 19 blocks, as indicated in figure 12b. The number and shape of these blocks is somewhat arbitrary, and these 19 blocks were drawn by hand. We provide 

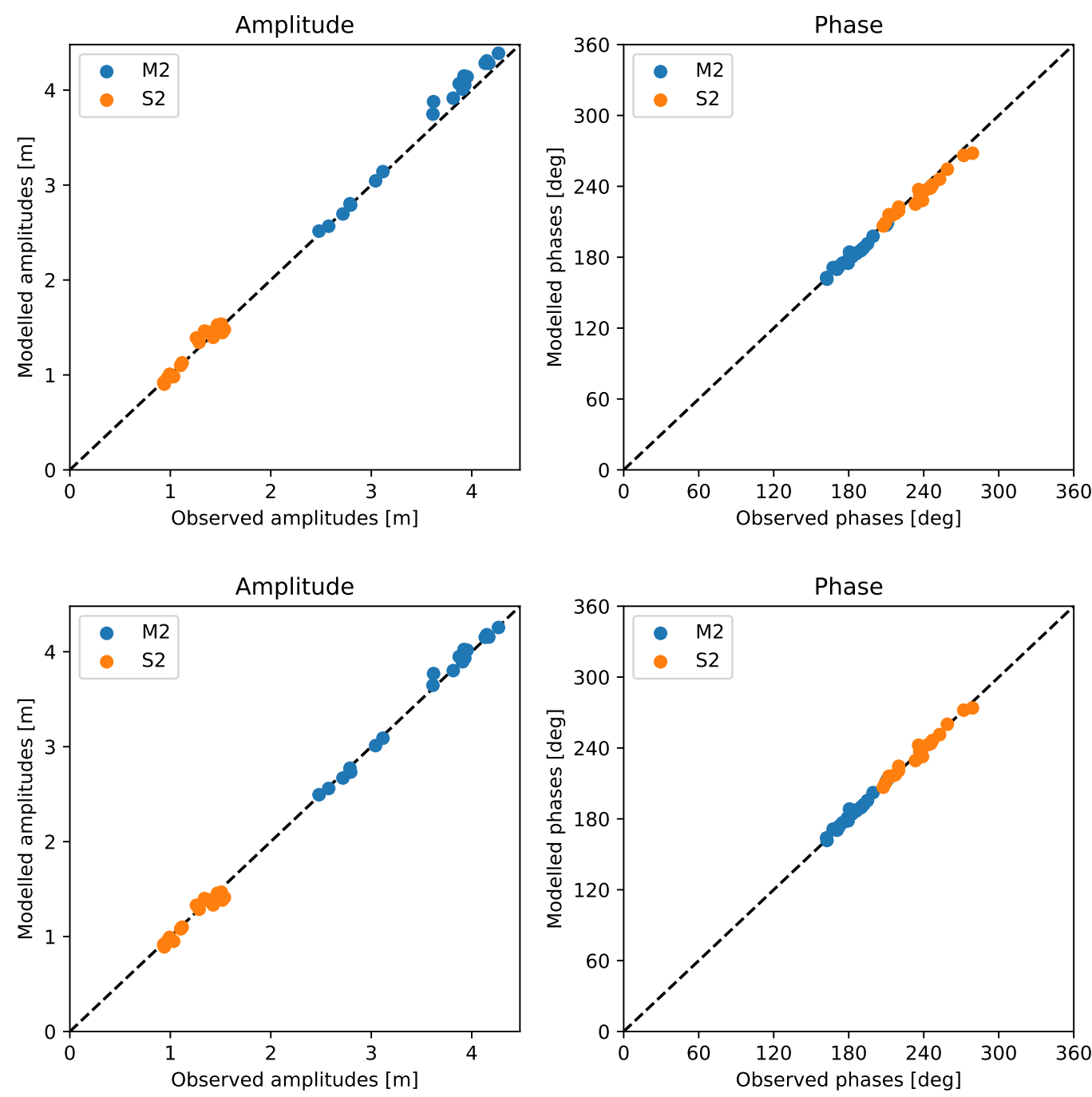

Figure 11: Scatter plots of modelled vs observed M2 and S2 amplitudes and phases in the Bristol Channel case study. Top: uncalibrated model with uniform friction coefficient $n=0.025 \mathrm{~s} \mathrm{~m}^{-1 / 3}$. Bottom: calibrated model, based on optimal $m=3$ experiment design. The root mean squared errors are $9.7 \mathrm{~cm}$ and $4.1^{\circ}$ before calibration, and $5.6 \mathrm{~cm}$ and $2.7^{\circ}$ after calibration.

a further constraint that groupings of these subdomains must produce contiguous blocks. For a grouping into $m=4$ parameters, this results in 31,865 possible experiment designs. Note that other possible approaches to parameterising the spatial variation of the bottom friction coefficient include the selection of independent points, between which the coefficient is determined by interpolation. The OED method can be trivially applied to any such space of experiment designs.

4. For each of the possible experiment designs, use the adjoint-derived gradients to compute the Fisher Information Matrix. Find the grouping which optimises a scalar measure of the FIM. Here we again use the modified Dcriterion as described above.

The optimal experiment design, following the above schema for a grouping into $m=4$ parameters, is shown in figure 13. The choice to use $m=4$ is again somewhat arbitrary, and used here purely as a demonstration of the method. Note the large variation in the size of the parameter regions, and the number of tide gauges included within 

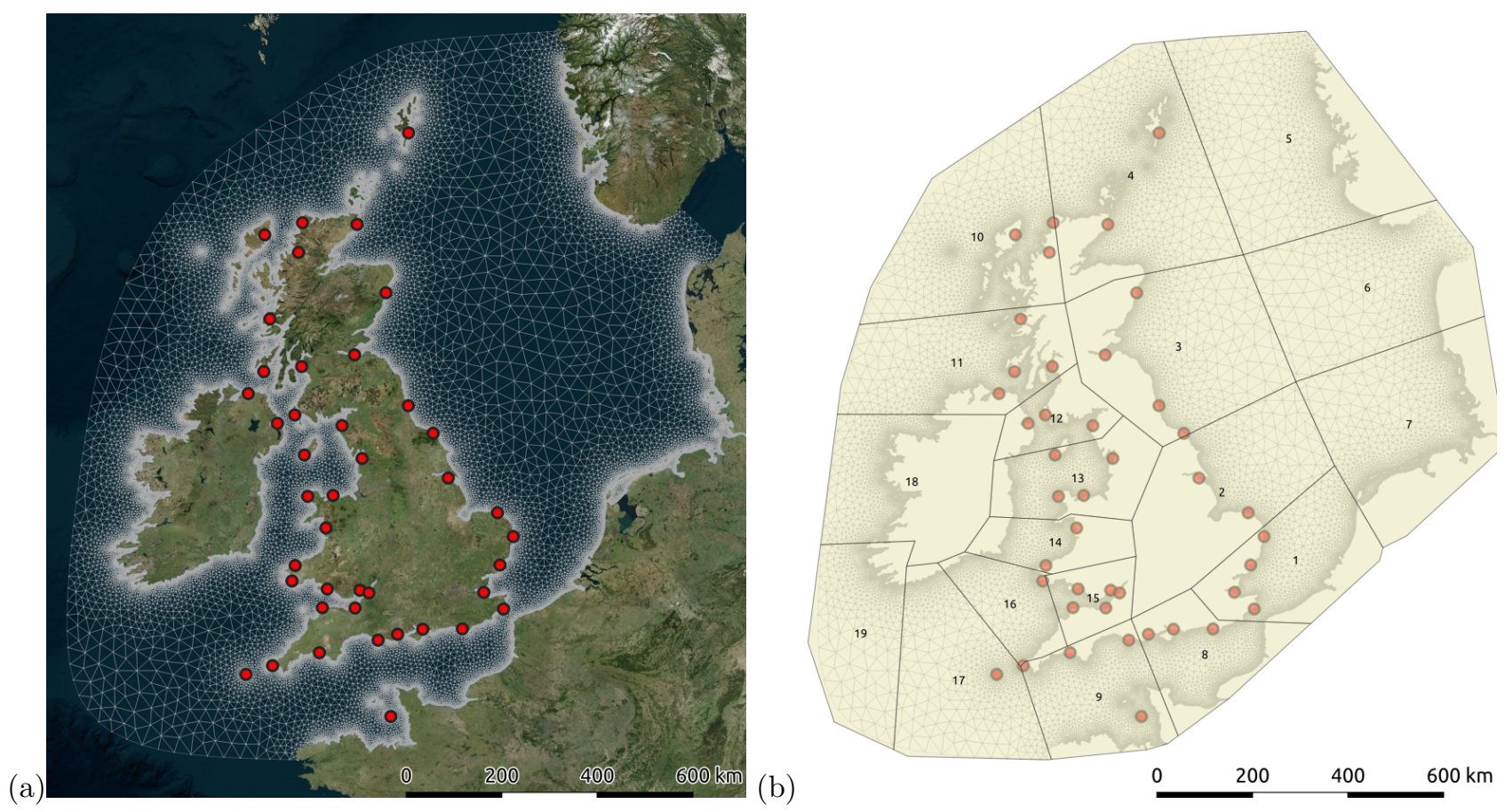

Figure 12: (a) Mesh used for continental shelf model. (b) Subdomains for friction parameterisation. The red circles indicate the locations of the tide gauges.

each. In particular, we find that the region including the North Sea covers by far the largest area, and contains a large number of tide gauges. Intuition might suggest that the optimal experiment design would likely consist of parameter regions of similar area, or containing similar volumes of observation data. However, a recent study on storm surge sensitivity to bottom friction coefficient in the North Sea (Warder et al., 2021) found that, due to the dynamics of surge propagation along the east coast of the UK, several tide gauge locations in the region exhibit similar spatial patterns of sensitivity to the bottom friction coefficient in the North Sea. This suggests that the tide gauges in the North Sea provide redundant information, which may explain why the optimal experiment design assigns a single friction parameter to the entire North Sea. In contrast, the dynamics within the Irish Sea are more complex, and the tide gauges in the region provide more diverse information about the bottom friction parameter, facilitating an experiment design featuring a greater number of friction parameters in the area.

\section{Discussion}

This study has demonstrated the application of an optimal experiment design procedure to the problem of bottom friction parameter estimation. In particular, we have addressed the problem that the spatial variation of the bottom friction parameter is unknown. The proposed OED method is able to identify a low-dimensional representation of the friction coefficient, resulting in parameters which can be constrained to a specified uncertainty by a given set of observations. We emphasise that the OED method is distinct from, and more useful than, the selection of a parameter space which can attain a specified agreement between model outputs and observations. A model calibration process motivated only by minimising the misfit between model outputs and observations would favour the use of a large number of tuning parameters, which can produce low model-observation misfits while uncertainty in the estimated parameters (and therefore in model outputs at new locations) remains high. A common technique 


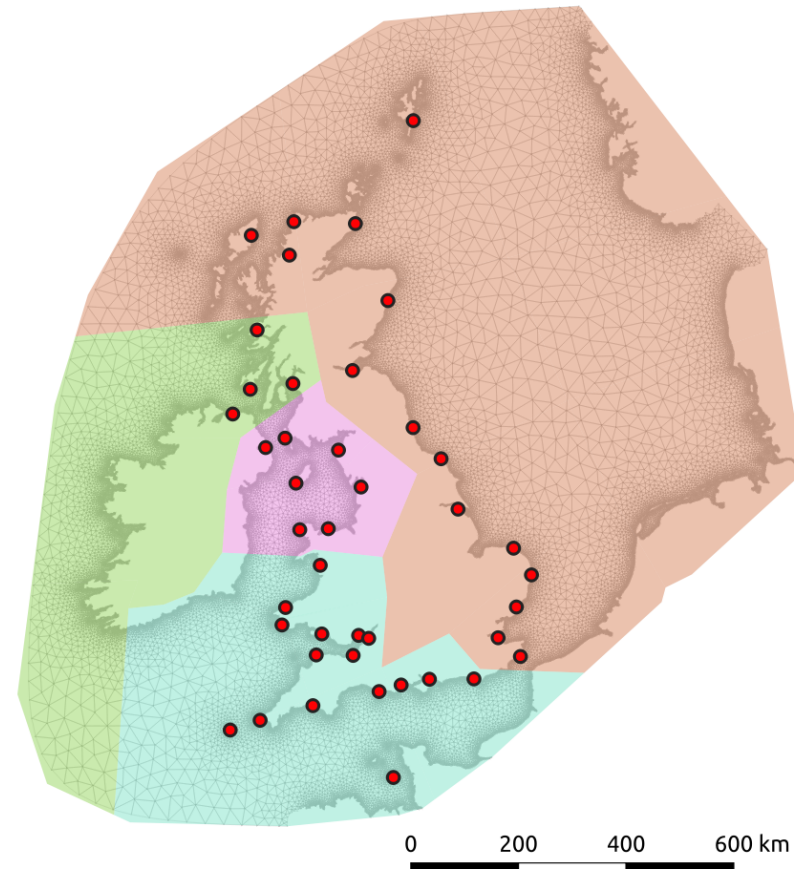

Figure 13: Optimal grouping of the subdomains into $m=4$ groups.

within the literature for addressing this issue is regularisation of the parameter estimation problem. The OED framework proposed within this paper offers an alternative approach, avoiding the need for regularisation. The OED approach constitutes a rigorous method for identifying a parameterisation of the unknown friction coefficient field which can be constrained to a specified precision by a given set of observations.

However, we note that it is not possible to interpret the OED-derived spatial pattern of the friction coefficient field as providing information relating to the underlying physical processes governing bottom friction. Similarly, the poorer performance of experiment designs based on the a priori use of sediment data compared with simple slicesubdomains described in sections 4.1 and 4.2 does not imply that the observations are inconsistent with the sediment data. This is because, in selecting the bottom friction coefficient as the only model calibration parameter, we are implicitly assuming that bottom friction is the only source of model uncertainty. However, in reality there exist a variety of sources of modelling errors, including other uncertain model inputs, the choice of governing equations, and model discretisation errors. Since the bottom friction parameterisation accounts for subgrid-scale bathymetric variation, the mesh resolution is likely to be a particularly influential modelling choice. Despite this issue, it is common practice to perform model calibration with respect to bottom friction alone. The presented OED approach is therefore a valuable preliminary step in a model calibration study, since a tighter constraint on a selected set of unknown parameters increases confidence in model outputs at new locations, even if the calibrated parameters are implicitly correcting for other modelling errors.

Our results indicate that the number of friction parameters that can be well constrained by the observations is typically much smaller than the number of observations. This is due in part to the ratio between the observation error variance and the target parameter estimate covariance (and the relationship between them via the model), but also due to redundancy in the information contained in the observations. This is particularly evident in the application to the northwest European continental shelf. In other words, a large quantity of data does not justify the estimation 
of a large number of model parameters, and the methods proposed within this work offer a rigorous technique for the selection of an appropriate set of model parameters for estimation from a given set of observations. The results of section 4.2 also demonstrate that the careful selection of the spatial distribution of the friction parameters (i.e. not just the number of parameters) is important. We have also found that, for the model domain considered, the choice to divide the domain by simple slices produces better-performing experiment designs than the use of sediment data to a priori constrain the spatial variation of the bottom friction coefficient. However, as noted above, this can be attributed to the relative sizes of the spaces of experiment designs for these choices, and does not necessarily imply that additional physical knowledge via sediment data cannot be beneficial within parameter estimation.

It is useful to compare the computational cost associated with the OED method presented here with the cost of a 'trial and error' approach where the parameter estimation problem is solved for a selection of candidate experiment designs. The adjoint model runs used here for the construction of the Jacobian were a one-off computational overhead. An adjoint run of the Thetis model used within this work takes around 2.4 times the wallclock time of a forward model run. The 80 pairs of forward and adjoint runs required therefore incurred a computational cost of around 270 forward model runs. In contrast, the Bayesian inference algorithm (via a Gaussian process emulator) required 30, 40 and 50 model runs for two-, three- and four-parameter experiment designs, respectively. For the problems considered here, therefore, the computational cost of the OED methodology is less than 10 times that of an individual parameter estimation experiment. Since the number of candidate designs considered within this study was of order 100, we therefore conclude that the adjoint-based OED method is computationally efficient compared with the trial-and-error approach. We note that the cost of the adjoint-based OED approach scales linearly with the number of observations used, and may therefore be poorly suited to an application to timeseries observations, or an extension to a large number of tidal constituents. However, as shown in section 4.3, for the right choice of $a$ priori constraint on the experiment design space, the optimal design itself shows low sensitivity to the precise set of observations used. An approach to adjoint-based OED for larger numbers of observations may therefore be to perform the OED based on a sample of the total observations; the results of this work suggest that the resulting experiment design may still be close to optimal, particularly if the observations contain significant redundant information. Furthermore, the computational cost of the adjoint-based approach scales well with the space of experiment designs. For applications where the number of observations is relatively small compared to the number of possible parameter space configurations, the adjoint-based OED procedure presented here is a highly efficient approach. For example, a relaxation of the relatively strong a priori constraints placed on the experiment designs within this work could significantly increase the number of possible designs, without having a significant impact on the computational cost of the study. The efficiency of the OED framework was also evident in the application to the northwest European continental shelf model, where a space of over 30,000 experiment designs was explored.

We note that an additional aspect relating to the computational cost of the OED approach of this work is the search algorithm used for finding the optimum within the space of possible experiment designs. Here, the selection of a priori constraints resulting in a fairly modest space of possible designs ensured that an exhaustive search algorithm was feasible. These a priori constraints also assisted in the interpretability of the optimal designs in terms of areas of the model domain, which can be a useful feature of parameter selection methods (Kravaris et al., 2013). However, the exploration of larger experiment design spaces will be considered in future work (in order to fully exploit the 
advantages of the adjoint-based approach as described above) and may require alternative optimisation approaches, such as genetic algorithms (e.g. Chu and Hahn (2007)), which have been used more commonly in the related problem of OED with respect to the set of observations (e.g. Catania and Paladino (2009)).

The use of the OED framework can also be compared with more traditional approaches in the literature, where low-dimensional parameterisations are selected less methodically. In such applications, regularisation (via a penalty term added to the misfit functional) is typically required in order to avoid an under-constrained calibration problem, where the observation data is insufficient to infer all of the unknown parameters. The use of the OED framework presented within this paper avoids the under-constrained problem entirely, and can be considered as an alternative to regularisation. The regularisation approach requires the selection of one or more regularisation parameters, controlling the magnitude of the penalty term in the functional. A cross-validation method for regularisation parameter selection, such as in Ullman and Wilson (1998), not only requires a second observation dataset for the cross-validation (whereas the OED framework does not), but also requires the repeated optimisation of the model with respect to the control parameters, for each possible choice of regularisation parameter, thus increasing the computational cost. The construction and solution of the OED problem within our Bristol Channel case study required 80 adjoint model runs (and note that these model runs can be performed simultaneously, i.e. the solution of the OED problem is "embarrassingly parallel"). A typical adjoint gradient-based approach to model calibration might require $\mathcal{O}(20)$ iterations to converge, and must be run several times for different regularisation parameter values. The OED approach is therefore competitive in terms of computational cost.

There are a number of avenues for the further application of OED methods within coastal ocean model parameter estimation. Firstly, we chose within this work to construct the experiment designs based on subdomains, within which the parameter is taken to be uniform. An alternative approach would be to use the OED framework to find independent points, between which the parameter is determined by interpolation. Secondly, the choice within this work to define a modified D-criterion to compare experiment designs has produced good results, but other design criteria are possible. For example, Machado et al. (2009) combine a normalised D-criterion with a modified E-criterion which characterises the shape of the parameter estimate confidence region, thus favouring designs producing similar constraints on all unknown parameters (i.e. a more spherical parameter confidence ellipsoid). Thirdly, a complementary application of OED within friction parameter estimation would be to identify an optimal observation strategy for constraining a given set of friction parameters. Integrated approaches to simultaneously optimise the observation strategy and the parameter space are also possible (Chu and Hahn, 2008), and may be valuable in coastal ocean applications. Fourthly, this study has considered only the estimation of uncertain bottom friction parameters. Although this is a common parameter for calibration within the numerical coastal ocean modelling literature, parameter estimation methods can also be applied to other model inputs including bathymetry (e.g. Mourre et al. (2004)), boundary conditions (e.g. Chen et al. (2014)), or combinations of multiple inputs simultaneously (e.g. Heemink et al. (2002)). The OED framework presented here is highly general and can be readily applied to these other sources of uncertainty. Note also that the computational cost of the OED methodology would not increase if additional model inputs were included. Finally, while model nonlinearity does not appear to have prevented the identification of 'good' experiment designs within this work, the application of the OED method to other model inputs, or combinations thereof, may require appropriate treatment of this nonlinearity, for example via maximin design (e.g. Ushijima and 
Yeh (2015)). Several of these aspects will be explored in future work.

\section{Conclusion}

In this study, we have applied an optimal experiment design technique to the identification of optimal piecewiseconstant representations of the Manning's $n$ coefficient within a numerical coastal ocean model. We have taken a region containing the Bristol Channel and Severn Estuary as a primary case study, where we have used harmonic analysis data at 20 locations within the domain for the estimation of the bottom friction parameter. We have further demonstrated the application of the framework to a model of the northwest European continental shelf, based on the assimilation of harmonic analysis data from 42 tide gauge locations. The key advantage of OED methods is that the experiment design (i.e. the observation strategy or the configuration of the input parameter space for model calibration) can be optimised in advance of the observations being made, or computational resources being applied to perform the model calibration itself. Here we used a method based on the Fisher Information Matrix, with the sensitivity of the model outputs with respect to the friction coefficient computed via a numerical adjoint model. As the optimality criterion, we proposed a modified D-criterion related to the determinant of the Fisher Information Matrix, motivated by achieving the tightest possible constraints on the estimated parameters, and avoiding the need for regularisation of the calibration problem.

The results of this study suggest that the modified D-criterion performs well in characterising the ability of a given experiment design to constrain the unknown friction parameters. This was verified by testing a variety of experiment designs within a Bayesian inference algorithm, and comparing the ModD criterion with an equivalent measure of the parameter covariance matrix returned by the Bayesian inference algorithm. The results show that the experiment design has a strong influence on the parameter constraints achievable from a given set of observation data. For example, the best experiment designs for the estimation of four parameters are able to provide tighter constraints on the unknown parameters than poor designs estimating only three parameters. This demonstrates the value of such an OED framework.

The framework also facilitates an investigation of the response of experiment design performance to the availability of observation data. For the observation dataset used for the Bristol Channel case study, consisting of M2 and S2 harmonic amplitudes and phases at 20 gauge locations, we find that the observations contain a significant amount of redundant information. The OED criterion initially increases rapidly as we increase the number of observation locations used, but subsequently flattens. Once this occurs, the parameter constraints can only be improved by a reduction in observation uncertainty, or the inclusion of additional types of observations, i.e. harmonic phases in addition to amplitudes, or additional harmonic constituents. This result has implications on strategies for observation surveys, and optimal experiment design with respect to observation strategy will be considered in future work.

Finally, while the computational cost of our approach depends on the volume of observation data, our results demonstrate that for practical parameter estimation problems the OED procedure can be considered a computationally efficient approach. For our case studies, the overall computational cost of the OED framework was on the order of 10 times that of performing an individual parameter estimation experiment with a given design. The OED framework is therefore not prohibitively expensive, compared with either a trial-and-error approach to finding the optimal experiment design, or a more traditional regularisation approach requiring repeated optimisations in order 
to select a regularisation parameter.

In summary, we have demonstrated that our OED framework can be used to formulate well-constrained calibration problems, and can be a valuable preliminary step in a parameter estimation study.

\section{Acknowledgements}

We acknowledge funding from EPSRC under grants EP/R029423/1, EP/R511547/1 as well as the EPSRC Centre for Doctoral Training in Fluid Dynamics across Scales (EP/L016230/1). We additionally acknowledge the Research Computing Service at Imperial College London for HPC resources, and thank Mariana Clare for helpful comments on the manuscript. This study uses data from the National Tidal and Sea Level Facility, provided by the British Oceanographic Data Centre and funded by the Environment Agency. 


\section{References}

T. A. A. Adcock, S. Draper, and T. Nishino. Tidal power generation-a review of hydrodynamic modelling. Proceedings of the Institution of Mechanical Engineers, Part A: Journal of Power and Energy, 229(7):755-771, 2015.

J. E. Alaña and C. Theodoropoulos. Optimal location of measurements for parameter estimation of distributed parameter systems. Computers \& chemical engineering, 35(1):106-120, 2011.

M. Altaf, M. Verlaan, and A. Heemink. Efficient identification of uncertain parameters in a large-scale tidal model of the european continental shelf by proper orthogonal decomposition. International Journal for Numerical Methods in Fluids, 68(4):422-450, 2012.

A. Angeloudis and R. A. Falconer. Sensitivity of tidal lagoon and barrage hydrodynamic impacts and energy outputs to operational characteristics. Renewable Energy, 114:337-351, 2017.

G. J. Arcement and V. R. Schneider. Guide for selecting manning's roughness coefficients for natural channels and flood plains, 1989 .

A. Avdis, A. S. Candy, J. Hill, S. C. Kramer, and M. D. Piggott. Efficient unstructured mesh generation for marine renewable energy applications. Renewable Energy, 116:842-856, 2018.

E. Balsa-Canto, A. A. Alonso, and J. R. Banga. Computational procedures for optimal experimental design in biological systems. IET systems biology, 2(4):163-172, 2008.

F. G. Blanchet, P. Legendre, and D. Borcard. Forward selection of explanatory variables. Ecology, 89(9):2623-2632, 2008.

F. Catania and O. Paladino. Optimal sampling for the estimation of dispersion parameters in soil columns using an iterative genetic algorithm. Environmental Modelling $\&$ Software, 24(1):115-123, 2009.

H. Chen, A. Cao, J. Zhang, C. Miao, and X. Lv. Estimation of spatially varying open boundary conditions for a numerical internal tidal model with adjoint method. Mathematics and Computers in Simulation, 97:14-38, 2014.

W.-B. Chen and W.-C. Liu. Investigating the fate and transport of fecal coliform contamination in a tidal estuarine system using a three-dimensional model. Marine Pollution Bulletin, 116(1-2):365-384, 2017.

Y. Chu and J. Hahn. Parameter set selection for estimation of nonlinear dynamic systems. AIChE journal, 53(11): $2858-2870,2007$.

Y. Chu and J. Hahn. Integrating parameter selection with experimental design under uncertainty for nonlinear dynamic systems. AIChE Journal, 54(9):2310-2320, 2008.

Y. Chu and J. Hahn. Parameter set selection via clustering of parameters into pairwise indistinguishable groups of parameters. Industrial \& Engineering Chemistry Research, 48(13):6000-6009, 2009.

S. Das and R. Lardner. On the estimation of parameters of hydraulic models by assimilation of periodic tidal data. Journal of Geophysical Research: Oceans, 96(C8):15187-15196, 1991. 
A. Davies and P. Robins. Residual flow, bedforms and sediment transport in a tidal channel modelled with variable bed roughness. Geomorphology, 295:855-872, 2017.

A. de Brauwere, F. De Ridder, O. Gourgue, J. Lambrechts, R. Comblen, R. Pintelon, J. Passerat, P. Servais, M. Elskens, W. Baeyens, et al. Design of a sampling strategy to optimally calibrate a reactive transport model: Exploring the potential for escherichia coli in the scheldt estuary. Environmental modelling 83 software, 24(8): 969-981, 2009.

Digimap. Marine Themes Digital Elevation Model 6 Arc Second [ASC geospatial data], Scale 1:250000, Tiles: 2052010080, 2052010060, 2052010040, 2050010100, 2050010080, 2050010060, 2050010040, 2048010100, 2048010080, 2048010060, 2048010040, Updated: 25 October 2013, OceanWise, Using: EDINA Marine Digimap Service, https://digimap.edina.ac.uk, Downloaded: 2020-07-14, 2013.

K. Döös, J. Nycander, and P. Sigray. Slope-dependent friction in a barotropic model. Journal of Geophysical Research: Oceans, 109(C1), 2004.

G. D. Egbert and S. Y. Erofeeva. Efficient inverse modeling of barotropic ocean tides. Journal of Atmospheric and Oceanic Technology, 19(2):183-204, 2002. doi: 10.1175/1520-0426(2002)019〈0183:EIMOBO〉2.0.CO;2.

A. F. Emery and A. V. Nenarokomov. Optimal experiment design. Measurement Science and Technology, 9(6):864, 1998.

P. E. Farrell, D. A. Ham, S. W. Funke, and M. E. Rognes. Automated derivation of the adjoint of high-level transient finite element programs. SIAM Journal on Scientific Computing, 35(4):C369-C393, 2013.

V. V. Fedorov. Theory of optimal experiments. 1972.

R. A. Flather. Existing operational oceanography. Coastal Engineering, 41(1-3):13-40, 2000.

I. Ford, D. Titterington, and C. P. Kitsos. Recent advances in nonlinear experimental design. Technometrics, 31(1): 49-60x, 1989.

C. Geuzaine and J. F. Remacle. Gmsh: A 3-D finite element mesh generator with built-in pre- and post-processing facilities. International Journal for Numerical Methods in Engineering, 79(11):1309-1331, 2009. doi: 10.1002/ nme.2579.

Z. Goss, D. Coles, and M. Piggott. Identifying economically viable tidal sites within the alderney race through optimization of levelized cost of energy. Philosophical Transactions of the Royal Society A, 378(2178):20190500, 2020.

GPy. GPy: A Gaussian process framework in python. http://github.com/SheffieldML/GPy, since 2012.

L. Graham, T. Butler, S. Walsh, C. Dawson, and J. J. Westerink. A Measure-Theoretic Algorithm for Estimating Bottom Friction in a Coastal Inlet: Case Study of Bay St. Louis during Hurricane Gustav (2008). Monthly Weather Review, 145:929-954, 2017. ISSN 0027-0644. doi: 10.1175/mwr-d-16-0149.1. 
N. Guillou and J. Thiébot. The impact of seabed rock roughness on tidal stream power extraction. Energy, 112: 762-773, 2016. doi: 10.1016/j.energy.2016.06.053.

J. W. Hall, L. J. Manning, and R. K. Hankin. Bayesian calibration of a flood inundation model using spatial data. Water Resources Research, 47(5):1-14, 2011. doi: 10.1029/2009WR008541.

W. K. Hastings. Monte Carlo sampling methods using Markov chains and their applications. 1970.

A. Heemink, E. Mouthaan, M. Roest, E. Vollebregt, K. Robaczewska, and M. Verlaan. Inverse 3d shallow water flow modelling of the continental shelf. Continental Shelf Research, 22(3):465-484, 2002.

P. Hristov, F. DiazDelaO, E. S. Flores, C. Guzmán, and U. Farooq. Probabilistic sensitivity analysis to understand the influence of micromechanical properties of wood on its macroscopic response. Composite Structures, 181: 229-239, 2017.

X. Huan and Y. M. Marzouk. Simulation-based optimal bayesian experimental design for nonlinear systems. Journal of Computational Physics, 232(1):288-317, 2013.

N. Huybrechts, H. Smaoui, S. Orseau, P. Tassi, and F. Klein. Automatic calibration of bed friction coefficients to reduce the influence of seasonal variation: Case of the gironde estuary. Journal of Waterway, Port, Coastal, and Ocean Engineering, 147(3):05021004, 2021.

T. Kärnä, B. De Brye, O. Gourgue, J. Lambrechts, R. Comblen, V. Legat, and E. Deleersnijder. A fully implicit wetting-drying method for dg-fem shallow water models, with an application to the scheldt estuary. Computer Methods in Applied Mechanics and Engineering, 200(5-8):509-524, 2011.

T. Kärnä, S. C. Kramer, L. Mitchell, D. A. Ham, M. D. Piggott, and A. M. Baptista. Thetis coastal ocean model: discontinuous galerkin discretization for the three-dimensional hydrostatic equations. Geoscientific Model Development, 11(11):4359-4382, 2018.

S. Kramer, T. Kärnä, J. Hill, and S. W. Funke. stephankramer/uptide: First release of uptide v1.0, 2020. http: //doi.org/10.5281/zenodo.3909652.

C. Kravaris, J. Hahn, and Y. Chu. Advances and selected recent developments in state and parameter estimation. Computers $\mathcal{E}$ chemical engineering, 51:111-123, 2013.

R. H. Langland. Issues in targeted observing. Quarterly Journal of the Royal Meteorological Society: A journal of the atmospheric sciences, applied meteorology and physical oceanography, 131(613):3409-3425, 2005.

R. Lardner, A. Al-Rabeh, and N. Gunay. Optimal estimation of parameters for a two-dimensional hydrodynamical model of the arabian gulf. Journal of Geophysical Research: Oceans, 98(C10):18229-18242, 1993.

R. Li, M. A. Henson, and M. J. Kurtz. Selection of model parameters for off-line parameter estimation. IEEE Transactions on control systems technology, 12(3):402-412, 2004.

X. Li, A. Plater, and N. Leonardi. Modelling the transport and export of sediments in macrotidal estuaries with eroding salt marsh. Estuaries and coasts, 41(6):1551-1564, 2018. 
X. Lu and J. Zhang. Numerical study on spatially varying bottom friction coefficient of a $2 \mathrm{~d}$ tidal model with adjoint method. Continental Shelf Research, 26(16):1905-1923, 2006.

C. Lyddon, J. M. Brown, N. Leonardi, and A. J. Plater. Uncertainty in estuarine extreme water level predictions due to surge-tide interaction. PloS one, 13(10):e0206200, 2018.

V. C. Machado, G. Tapia, D. Gabriel, J. Lafuente, and J. A. Baeza. Systematic identifiability study based on the fisher information matrix for reducing the number of parameters calibration of an activated sludge model. Environmental Modelling \& Software, 24(11):1274-1284, 2009.

L. Mackie, D. Coles, M. Piggott, and A. Angeloudis. The Potential for Tidal Range Energy Systems to Provide Continuous Power: A UK Case Study. Journal of Marine Science and Engineering, 8(10):780, 2020a.

L. Mackie, P. S. Evans, M. J. Harrold, T. O’Doherty, M. D. Piggott, and A. Angeloudis. Modelling hydrodynamics in an energetic tidal strait with pronounced bathymetric features. In review, submitted to Applied Ocean Research, 2020b. doi: https://doi.org/10.31223/osf.io/8txmd.

K. N. Marshall, I. C. Kaplan, E. E. Hodgson, A. Hermann, D. S. Busch, P. McElhany, T. E. Essington, C. J. Harvey, and E. A. Fulton. Risks of ocean acidification in the california current food web and fisheries: ecosystem model projections. Global Change Biology, 23(4):1525-1539, 2017.

S. Maßmann. Tides on unstructured meshes. PhD thesis, Universitat Bremen, 2010.

T. Mayo, T. Butler, C. Dawson, and I. Hoteit. Data assimilation within the Advanced Circulation (ADCIRC) modeling framework for the estimation of Manning's friction coefficient. Ocean Modelling, 76:43-58, 2014. ISSN 14635003. doi: 10.1016/j.ocemod.2014.01.001. URL http://dx.doi.org/10.1016/j.ocemod.2014.01.001.

S. K. Mitusch, S. W. Funke, and J. S. Dokken. dolfin-adjoint 2018.1: automated adjoints for FEniCS and Firedrake. Journal of Open Source Software, 4(38), 2019.

B. Mourre, P. De Mey, F. Lyard, and C. Le Provost. Assimilation of sea level data over continental shelves: an ensemble method for the exploration of model errors due to uncertainties in bathymetry. Dynamics of atmospheres and oceans, 38(2):93-121, 2004.

S. P. Neill, A. Angeloudis, P. E. Robins, I. Walkington, S. L. Ward, I. Masters, M. J. Lewis, M. Piano, A. Avdis, M. D. Piggott, et al. Tidal range energy resource and optimization-past perspectives and future challenges. Renewable energy, 127:763-778, 2018.

W. Pan, S. C. Kramer, T. Kärnä, and M. D. Piggott. Comparing non-hydrostatic extensions to a discontinuous finite element coastal ocean model. Ocean Modelling, 151:101634, 2020. ISSN 1463-5003. doi: https://doi.org/10.1016/ j.ocemod.2020.101634. URL http://www.sciencedirect.com/science/article/pii/S1463500319303221.

A. Pepelyshev, V. B. Melas, N. Strigul, and H. Dette. Design of experiments for the monod model: robust and efficient designs. Technical report, Technical Report, 2004. 
R. Periáñez, M. Casas-Ruíz, and J. Bolívar. Tidal circulation, sediment and pollutant transport in cádiz bay (sw spain): a modelling study. Ocean engineering, 69:60-69, 2013.

C. E. Rasmussen. Gaussian processes in machine learning. In Summer School on Machine Learning, pages 63-71. Springer, 2003.

F. Rathgeber, D. A. Ham, L. Mitchell, M. Lange, F. Luporini, A. T. McRae, G.-T. Bercea, G. R. Markall, and P. H. Kelly. Firedrake: automating the finite element method by composing abstractions. ACM Transactions on Mathematical Software (TOMS), 43(3):1-27, 2016.

C. R. Rojas, J. S. Welsh, G. C. Goodwin, and A. Feuer. Robust optimal experiment design for system identification. Automatica, 43(6):993-1008, 2007.

SHOM, 2013. URL https://data.shom.fr. Accessed 2019-04-10.

I. M. Sobol. Global sensitivity indices for nonlinear mathematical models and their Monte Carlo estimates. Mathematics and Computers in Simulation, 55(1-3):271-280, 2001.

T. Söderström and P. Stoica. System identification. Prentice-Hall International, 1989.

I. Sraj, M. Iskandarani, A. Srinivasan, W. C. Thacker, J. Winokur, A. Alexanderian, C. Y. Lee, S. S. Chen, and O. M. Knio. Bayesian inference of drag parameters using AXBT data from typhoon fanapi. Monthly Weather Review, 141(7):2347-2367, 2013. doi: 10.1175/MWR-D-12-00228.1.

I. Sraj, M. Iskandarani, W. C. Thacker, A. Srinivasan, and O. M. Knio. Drag parameter estimation using gradients and hessian from a polynomial chaos model surrogate. Monthly Weather Review, 142(2):933-941, 2014a.

I. Sraj, K. T. Mandli, O. M. Knio, C. N. Dawson, and I. Hoteit. Uncertainty quantification and inference of Manning's friction coefficients using DART buoy data during the Tōhoku tsunami. Ocean Modelling, 83:82-97, 2014b. doi: 10.1016/j.ocemod.2014.09.001.

N. Strigul, H. Dette, and V. B. Melas. A practical guide for optimal designs of experiments in the monod model. Environmental Modelling \&3 Software, 24(9):1019-1026, 2009.

A. Y. Sun. A robust geostatistical approach to contaminant source identification. Water Resources Research, 43(2), 2007.

D. Ucinski. Optimal measurement methods for distributed parameter system identification. CRC press, 2004.

D. S. Ullman and R. E. Wilson. Model parameter estimation from data assimilation modeling: Temporal and spatial variability of the bottom drag coefficient. Journal of Geophysical Research: Oceans, 103(C3):5531-5549, 1998.

T. T. Ushijima and W. W. Yeh. Experimental design for estimating unknown hydraulic conductivity in an aquifer using a genetic algorithm and reduced order model. Advances in Water Resources, 86:193-208, 2015.

V. Vandenberghe, A. van Griensven, and W. Bauwens. Detection of the most optimal measuring points for water quality variables: application to the river water quality model of the river dender in eswat. Water science and technology, 46(3):1-7, 2002. 
C. V. Vouriot, A. Angeloudis, S. C. Kramer, and M. D. Piggott. Fate of large-scale vortices in idealized tidal lagoons. Environmental Fluid Mechanics, 19(2):329-348, 2019.

É. Walter and L. Pronzato. Qualitative and quantitative experiment design for phenomenological models - a survey. Automatica, 26(2):195-213, 1990.

D. Wang, A. Cao, J. Zhang, D. Fan, Y. Liu, and Y. Zhang. A three-dimensional cohesive sediment transport model with data assimilation: Model development, sensitivity analysis and parameter estimation. Estuarine, Coastal and Shelf Science, 206:87-100, 2018.

T. Wang and Z. Yang. A tidal hydrodynamic model for cook inlet, alaska, to support tidal energy resource characterization. Journal of Marine Science and Engineering, 8(4):254, 2020.

S. C. Warder, K. J. Horsburgh, and M. D. Piggott. Understanding the contribution of uncertain wind stress to storm surge predictions. In 4th IMA International Conference on Flood Risk, Swansea, 2019.

S. C. Warder, K. J. Horsburgh, and M. D. Piggott. Adjoint-based sensitivity analysis for a numerical storm surge model. Ocean Modelling, 160:101766, 2021.

P. Wessel and W. H. F. Smith. A global, self-consistent, hierarchical, high-resolution shoreline database. Journal of Geophysical Research: Solid Earth, 101(B4):8741-8743, 1996. doi: 10.1029/96JB00104.

P. Whomersley, J. Van der Molen, D. Holt, C. Trundle, S. Clark, and D. Fletcher. Modeling the dispersal of spiny lobster (palinurus elephas) larvae: Implications for future fisheries management and conservation measures. Frontiers in Marine Science, 5:58, 2018.

H. Yu, H. Yue, and P. Halling. Comprehensive experimental design for chemical engineering processes: A two-layer iterative design approach. Chemical Engineering Science, 189:135-153, 2018.

J. Zhang, X. Lu, P. Wang, and Y. P. Wang. Study on linear and nonlinear bottom friction parameterizations for regional tidal models using data assimilation. Continental Shelf Research, 31(6):555-573, 2011. 


\section{Appendix A. Bayesian inference}

Bayesian inference is a powerful statistical technique for solving inverse problems, and has been applied to bottom friction parameter estimation previously (Hall et al., 2011, Sraj et al., 2014b). Within this work, we use Bayesian inference to solve parameter estimation problems for a selected number of experiment designs. Our Bayesian inference framework proceeds via a Markov Chain Monte Carlo (MCMC) method, using a Gaussian process emulator (GPE) as a surrogate for the full numerical model, since the method relies on a large number of model evaluations. Appendix A.1 describes Gaussian process emulation, and Appendix A.2 details the MCMC algorithm.

\section{Appendix A.1. Gaussian process emulation}

This exposition follows Rasmussen (2003), to which the reader is referred for further detail. The crux of Gaussian process emulation is that, under the assumption that model outputs follow a multivariate Gaussian distribution, a vector of 'test' model outputs $\mathbf{f}_{*}$ for model inputs $\mathbf{X}_{*}$ can be predicted from 'training' model outputs $\mathbf{f}$ computed for model inputs $\mathbf{X}$. The model outputs satisfy the conditional distribution

$$
\mathbf{f}_{*} \mid \mathbf{f} \sim \mathcal{N}\left(\mu\left(\mathbf{X}_{*}\right)+\Sigma_{*}^{T} \Sigma^{-1}(\mathbf{f}-\mu(\mathbf{X})), \Sigma_{* *}-\Sigma_{*}^{T} \Sigma^{-1} \Sigma_{*}\right),
$$

where $\mu$ denotes the mean function, $\Sigma$ the covariance matrix for the training points, $\Sigma_{* *}$ the covariance matrix for the test points, and $\Sigma_{*}$ the covariance between the training and test points. The covariance matrices are typically parameterised by

$$
\Sigma=k(\mathbf{X}, \mathbf{X}) ; \Sigma_{*}=k\left(\mathbf{X}, \mathbf{X}_{*}\right) ; \Sigma_{* *}=k\left(\mathbf{X}_{*}, \mathbf{X}_{*}\right),
$$

where $k\left(x, x^{\prime}\right)$ is a covariance function, with a common choice given by

$$
k\left(x, x^{\prime}\right)=\sigma^{2} C\left(x, x^{\prime}\right),
$$

where $\sigma^{2}$ is a covariance parameter and $C\left(x, x^{\prime}\right)$ a parameterised correlation function. Similarly, the mean function $\mu$ is typically taken as a simple linear or quadratic function of the model inputs. The parameters introduced by the mean and covariance functions can be determined via maximum likelihood estimation using the training data. Once these parameters are determined, equation (A.1) can be evaluated at low computational cost, and the mean of the conditional distribution estimates model outputs for unseen values of the model inputs. The generalisation to higher dimensions and multiple model outputs is straightforward. Within this work, we use the Python package GPy (since 2012); the reader is referred to the package documentation for further implementation detail.

For the application to Bayesian inference within this study, the model inputs are the vector of unknown parameters $\boldsymbol{\theta}$ for a given experiment design, and the model outputs are the M2 and S2 amplitudes and phases at each of the 20 tide gauge locations (a total of 80 outputs). The training input samples ( $\mathbf{X}$ in the above notation) are selected using Latin Hypercube Sampling from uniform prior distributions in the range [0.01,0.05] for each Manning coefficient. According to the '10d' rule (Sobol, 2001, Hristov et al., 2017), it is common to train a Gaussian process emulator using at least $10 d$ samples, where $d$ is the number of input parameters. As a cautious approach, within this work we use $10(d+1)$ training samples for each selected experiment design to be tested using Bayesian inference. The corresponding output for each training sample is generated from a forward run of the Thetis numerical model comprising a 13-day spin-up period followed by a 14.77-day harmonic analysis period. Harmonic analysis for the M2 
and S2 constituents is performed at each of the 20 tide gauge locations, using the Python package uptide (Kramer et al., 2020). These outputs are used as the training data to construct a Gaussian process emulator as described above.

Prior to the use of the GPEs within the Bayesian inference algorithm, we first verified their faithfulness as a surrogate for the full numerical model by comparing their outputs with additional full numerical model runs for random samples of the model parameters. We found that the GPE error covariance calculated via equation (A.1) provides a good estimate of the true emulator error covariance, and that this covariance is small compared with the assumed observation uncertainty. This covariance is therefore neglected when using the GPE within the Bayesian inference algorithm.

\section{Appendix A.2. Markov Chain Monte Carlo algorithm}

The Bayesian inference framework follows a similar approach to Sraj et al. (2013, 2014b). Denoting the vector of tidal harmonic observations $\mathbf{y}$ and the vector of model parameters $\boldsymbol{\theta}$, Bayes' theorem gives

$$
\Pi(\boldsymbol{\theta} \mid \mathbf{y}) \propto L(\mathbf{y} \mid \boldsymbol{\theta}) q(\boldsymbol{\theta})
$$

where $\Pi$ is the posterior distribution of the parameters $\boldsymbol{\theta}$ given the observed data $\mathbf{y}, L$ is the likelihood of observing the outputs $\mathbf{y}$ given the parameters $\boldsymbol{\theta}$, and $q$ is the prior distribution of the parameter vector $\boldsymbol{\theta}$. The prior distribution on each individual Manning coefficient $\theta_{i}$ is taken as uniform in the range [0.01,0.05], hence

$$
q(\boldsymbol{\theta})= \begin{cases}\left(\frac{1}{0.05-0.01}\right)^{m} & \text { if } 0.01<\theta_{i}<0.05 \forall i \\ 0 & \text { otherwise. }\end{cases}
$$

It is assumed that the model-observation discrepancies $\mathbf{y}-\mathbf{f}$, where $\mathbf{f}$ is the vector of model outputs, are independent and identically distributed variables with zero mean, and a covariance matrix

$$
\Sigma_{\text {obs }}=\left(\begin{array}{cc}
\sigma_{\mathrm{amp}}^{2} \mathbb{I}_{40 \times 40} & 0 \\
0 & \sigma_{\text {phase }}^{2} \mathbb{I}_{40 \times 40}
\end{array}\right),
$$

where $\sigma_{\text {amp }}^{2}$ and $\sigma_{\text {phase }}^{2}$ are the amplitude and phase measurement covariances, respectively, as described in section 2.2, and II denotes an identity matrix of the specified size (we use 40 amplitude observations and 40 phase observations). The likelihood $L(\mathbf{y} \mid \boldsymbol{\theta})$ is therefore given by

$$
L(\mathbf{y} \mid \boldsymbol{\theta})=(2 \pi)^{-K / 2}\left|\Sigma_{o b s}\right|^{-1 / 2} \exp \left(-\frac{1}{2}(\mathbf{y}-\mathbf{f})^{T} \Sigma_{o b s}^{-1}(\mathbf{y}-\mathbf{f})\right),
$$

where $K=80$ is the number of observations.

Equation (A.4) gives the probability distribution of the unknown Manning coefficients, given the set of observations $\mathbf{y}$, and its evaluation represents the parameter estimation problem. A technique for sampling this posterior distribution when it cannot be directly calculated is the Markov Chain Monte Carlo (MCMC) method, which has the advantage that the constant of proportionality need not be determined. This work uses an implementation of the Random Walk Metropolis Hastings MCMC algorithm (Hastings, 1970), which is given by algorithm 1. Within the algorithm, we set the proposal distribution covariance matrix to

$$
\Sigma_{\text {step }}=0.001^{2} \mathbb{I}_{m \times m}
$$




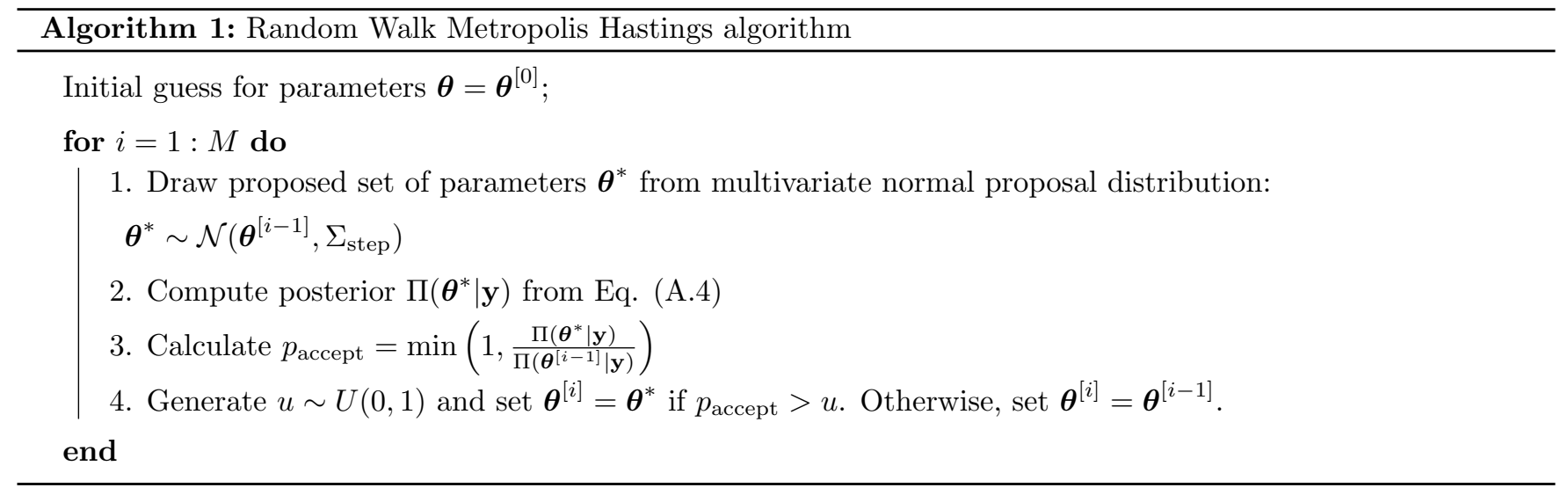

